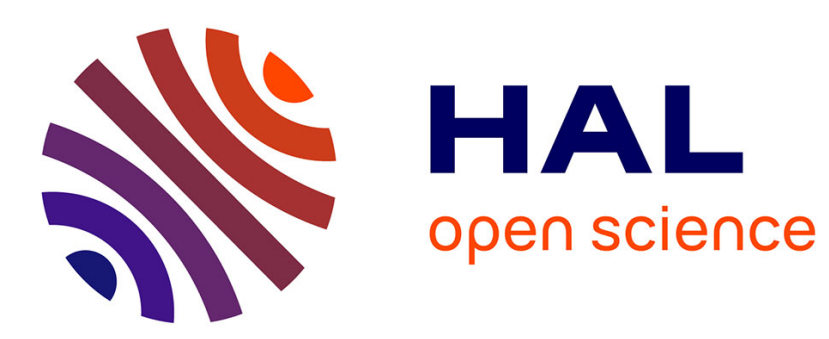

\title{
Time-domain simulation of wave propagation across resonant meta-interfaces
}

\author{
Marie Touboul, Bruno Lombard, Cédric Bellis
}

\section{To cite this version:}

Marie Touboul, Bruno Lombard, Cédric Bellis. Time-domain simulation of wave propagation across resonant meta-interfaces. Journal of Computational Physics, 2020, 414 (1). hal-02185076v2

\section{HAL Id: hal-02185076 \\ https://hal.science/hal-02185076v2}

Submitted on 11 Apr 2020

HAL is a multi-disciplinary open access archive for the deposit and dissemination of scientific research documents, whether they are published or not. The documents may come from teaching and research institutions in France or abroad, or from public or private research centers.
L'archive ouverte pluridisciplinaire HAL, est destinée au dépôt et à la diffusion de documents scientifiques de niveau recherche, publiés ou non, émanant des établissements d'enseignement et de recherche français ou étrangers, des laboratoires publics ou privés. 


\title{
Time-domain simulation of wave propagation across resonant meta-interfaces
}

\author{
Marie Touboul $^{\mathrm{a}}$, Bruno Lombard ${ }^{\mathrm{a}, *}$, Cédric Bellis ${ }^{\mathrm{a}}$ \\ ${ }^{a}$ Aix Marseille Univ, CNRS, Centrale Marseille, LMA UMR 7031, Marseille, France
}

\begin{abstract}
Meta-interface models stem from the homogenization, in a low-frequency dynamic regime, of thin heterogeneous layers that are structured to achieve uncommon properties at the macroscopic level. When the layer is composed of a thin periodic array of highly-contrasted inclusions embedded within a homogeneous background medium then the corresponding effective interface model is characterized by jump conditions that, in the harmonic regime, involve some singular frequency-dependent terms. In this context, the article is concerned with the simulation of transient waves across such resonant meta-interfaces and a numerical method is proposed to handle the associated resonant jump conditions. To do so, a set of auxiliary variables is introduced locally along the interface and an augmented system of first-order equations in time accompanied with local-in-time jump conditions is derived. This system is then discretized on a Cartesian grid and solved using a high-order finite-difference scheme while the complexity associated with the geometry of the interface and the jump conditions is handled using an immersed interface method. A set of numerical examples in 1D and 2D is proposed to illustrate and validate the overall numerical approach, and quantitative comparisons with semi-analytical solutions are also provided.
\end{abstract}

Keywords: resonant microstructures, resonant effective interfaces, frequency-dependent jump conditions, immersed interface method, ADER scheme.

\section{Introduction}

Modeling and simulating the wave propagation within a microstructured medium can be handled efficiently by upscaling approaches such as the common homogenization methods $[2$, 23]. When the characteristic length-scale of the heterogeneities, which coincides with the period length in the case of periodic composite, is much smaller than a reference wavelength then the microstructure can be replaced by a homogeneous effective medium whose constitutive properties stem from those of the former. In the case where the micro-scale heterogeneities form a thin microstructured layer, see Figure 1, then dedicated homogenization methods must be employed [19] to derive effective jump conditions on an equivalent interface [21, 8, 7, 20, 4]. As a result of such a homogenization process, and owing to energy-based considerations, the heterogeneous layer is substituted by an enlarged interface, in which no calculation is performed

*Corresponding author. Tel.: +3349184524253.

Email addresses: touboul@lma.cnrs-mrs.fr (Marie Touboul), lombard@lma.cnrs-mrs.fr (Bruno Lombard), bellis@lma.cnrs-mrs.fr (Cédric Bellis) 
and no field is defined but on both sides of which effective jump conditions apply. The latter depond on the material and geometrical properties of the original microstructured layer and those of the matrix.

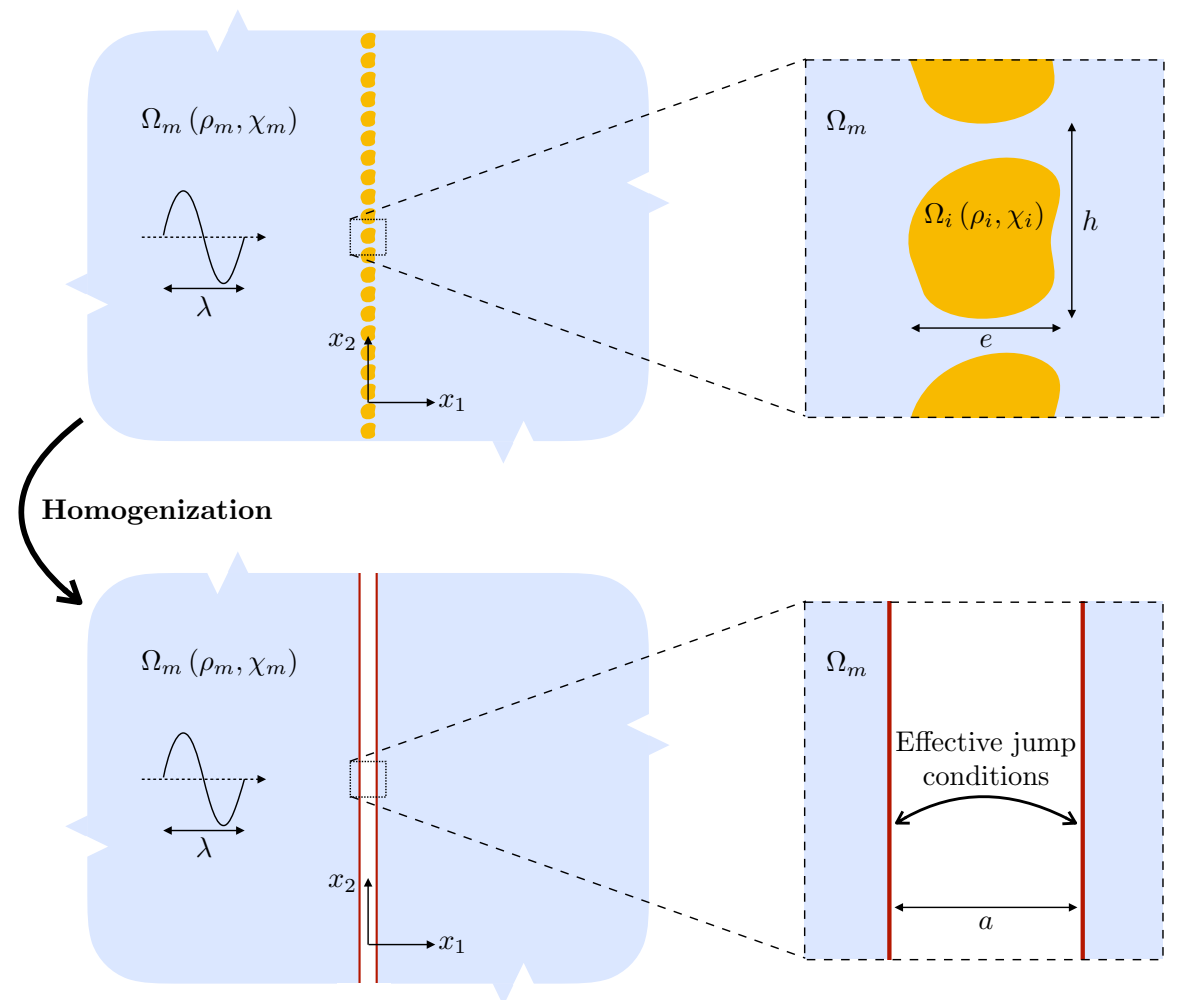

Figure 1: Homogenization process for a single periodic array of inclusions. (top) Original configuration with a thin microstructured layer, (bottom) Homogenized interface model.

In the case where the heterogeneous layer is composed of inclusions characterized by a large material contrast, relatively to the properties of the surrounding matrix, then illumination by an incident wave or an external source can give rise to internal resonances within the microstructure. By applying a suitable homogenization process to such a configuration, see [22], then this phenomenon can be modeled at the macroscopic level by effective jump conditions that, in the harmonic regime, are frequency-dependent. The occurence of such internal resonances, along with the associated homogenized models, offers new possibilities to control waves at the macroscopic level. This corresponds to the current paradigm of metamaterials and, in the present case, of resonant metasurfaces or meta-interfaces. As an example, resonant metasurfaces can be designed to optimize sound absorption [18, 24]. Exotic behaviors can also be sought and exploited, see e.g. the splitting of P- and SV-waves through elastic resonant metasurfaces [26].

In this context, the article aims at building a numerical method to simulate the interaction of transient waves with such resonant meta-interfaces. By opposition with frequency-domain approaches, time-domain formulations have two main advantages: i) they allow to simulate wide-band wave phenomena in a single computation; ii) they pave the way to problems involving nonlinearities, for which a monochromatic forcing requires to solve numerous Helmholtz equations due to the generation of harmonics. To perform such time-domain simulations, highorder finite difference schemes on uniform Cartesian grid are chosen for computational efficiency 
and accuracy. However, a straightforward discretization of the interfaces suffers classicaly from a number of drawbacks: errors of order one are introduced if the interfaces do not coincide with the mesh grid, naive stair-step discretizations yield spurious diffractions and jump conditions are not properly handled. To prevent from these drawbacks, the so-called immersed interface methods have shown to constitute efficient approaches [28]. Previous works have concerned the development of the Explicit Simplified Interface Method (ESIM) and its application to a variety of wave propagation problems $[15,6,16,13,11]$. In particular, simulation of time-domain acoustic wave propagation across enlarged effective interfaces have been previously addressed in [12] but in the case of non-resonant effective jump conditions. In the present study, the central question therefore concerns the consideration of resonant effective jump conditions that are frequency-dependent in the harmonic regime along with their implementation for wave propagation simulations in the time domain.

Handling resonant meta-interfaces introduces a number of difficulties compared to the case of non-resonant interfaces. The effective jump conditions associated with the former are indeed characterized by frequency-dependent terms in the harmonic regime that yield a convolution product when transposed in the time domain. Their implementation would therefore require to store the entire history of the traces of the solution along the enlarged interface while the computation of the time convolution integral itself would substantially increase the computational cost in a naive extension of the ESIM. As a consequence, it is required to develop a specific approach to handle efficiently this type of resonant interface models numerically. For this purpose, the auxiliary variables formalism, which has been previously employed in [1] in the case of bulk dispersive metamaterials in acoustics, is adapted here to deal with the resonant meta-interfaces considered. In particular, a set of auxiliary variables is introduced locally along the enlarged interface to handle the resonant behavior of the wavefield. Due to the coupling of these additional variables with the original solution fields of the wave equation, the proposed approach involves substantial modifications of the ESIM. In counterpart, the resulting computational performances of the proposed approach is comparable with those obtained in the reference case of non-resonant interfaces.

The article is organized as follows. In Section 2, the model associated with the resonant meta-interfaces considered is presented in acoustics. The auxiliary variables formalism is then introduced and implemented to obtain an equivalent augmented system of first-order equations in time with transformed jump conditions that are local in time. Section 3 focuses in detail on a numerical implementation in 1D: the fourth-order finite-difference scheme ADER is used in combination with the ESIM. A local error analysis is performed and sufficient conditions under which a given order of accuracy can be obtained are stated. Then Section 4 provides an overview of the extension of the proposed numerical method in 2D. Numerical experiments in 1D and 2D are finally presented in Section 5, while comparisons with semi-analytical solutions allow to assess quantitatively the performance of the approach developed. Comparisons with nonresonant jump conditions are also given to investigate the effects associated with the resonant behavior of the meta-interfaces considered.

\section{Physical model}

\subsection{Governing equations for resonant meta-interfaces}

Consider the propagation of acoustic waves in a background matrix $\Omega_{m} \subset \mathbb{R}^{2}$ that contains an infinite array of inclusions $\Omega_{i}$ arranged periodically with a spacing $h$, see Figure 1 . The media 
considered are assumed to be homogeneous and isotropic so that their constitutive parameters, being the mass density $\rho$ and the compressibility $\chi$, are piecewise constant:

$$
\rho(\boldsymbol{x})=\rho_{m} \mathbf{1}_{\Omega_{m}}(\boldsymbol{x})+\rho_{i} \mathbf{1}_{\Omega_{i}}(\boldsymbol{x}) \quad \text { and } \quad \chi(\boldsymbol{x})=\chi_{m} \mathbf{1}_{\Omega_{m}}(\boldsymbol{x})+\chi_{i} \mathbf{1}_{\Omega_{i}}(\boldsymbol{x})
$$

for all $\boldsymbol{x}=\left(x_{1}, x_{2}\right) \in \mathbb{R}^{2}$ and with $\mathbf{1}_{\mathcal{D}}$ being the indicator function of a given domain $\mathcal{D}$. Note that the compressibility parameter $\chi$ is related to the classical wave velocity $c$ by the relation $\chi=\left(\rho c^{2}\right)^{-1}$. In the frequency-domain, the governing equations for the acoustic velocity and pressure fields, denoted as $\boldsymbol{v}=\left(v_{1}, v_{2}\right)$ and $p$ respectively, read:

$$
\left\{\begin{array}{l}
\mathrm{i} \omega \rho(\boldsymbol{x}) \hat{\boldsymbol{v}}(\boldsymbol{x}, \omega)=-\nabla \hat{p}(\boldsymbol{x}, \omega), \\
\mathrm{i} \omega \chi(\boldsymbol{x}) \hat{p}(\boldsymbol{x}, \omega)=-\operatorname{div} \hat{\boldsymbol{v}}(\boldsymbol{x}, \omega),
\end{array}\right.
$$

where $\omega$ is the angular frequency and $\hat{f}$ being the Fourier transform of a field $f$. Moreover, $\hat{p}$ and $\hat{\boldsymbol{v}} \cdot \boldsymbol{\nu}$ are assumed to be continuous at the matrix/inclusion interfaces $\partial \Omega_{i}$ with normal $\boldsymbol{\nu}$. The equations (2) have to be completed with initial conditions and proper radiation conditions at infinity to ensure well-posedness. External sources terms may also be considered. Note that the system (2) is here considered in the acoustic case but it is also relevant to other physical configurations, such as linear anti-plane elasticity for which the fields $\boldsymbol{v}, p$ and the parameters $\chi$, $1 / \rho$ would stand instead for stress vector, velocity, mass density and shear modulus, respectively.

In this setting and defining the background wave velocity $c_{m}=1 / \sqrt{\rho_{m} \chi_{m}}$, it is further assumed that the characteristic length-scale $h$ of the microstructure is small compared to the wavelength $\lambda=2 \pi c_{m} / \omega$ within the matrix, i.e. $h \ll \lambda$, a condition which places the physical configuration considered in the low-frequency homogenization regime. For some values of the compressibility and the mass density, the wavelength within an inclusion is of order $h$. For a particular scaling between material parameters, the original problem defined above can be replaced by a resonant homogenized model that consists in a set of resonant effective jump conditions as shown in [22]. These conditions apply on an enlarged straight interface of width $a$, see Figure 1, and within which no calculation is performed and no field is defined. In the non-resonant case, it has been shown in [20] using an energy-based analysis that the width $a$ of the enlarged interface must be at least equal to the width $e$ of the microstructure. We follow the same criterion here, which leads, for an enlarged interface centered at $x_{1}=0$, to the model considered here:

$$
\begin{cases}\mathrm{i} \omega \rho_{m} \hat{\boldsymbol{v}}(\boldsymbol{x}, \omega)=-\boldsymbol{\nabla} \hat{p}(\boldsymbol{x}, \omega) & \left(\left|x_{1}\right| \geq a / 2, x_{2} \in \mathbb{R}\right) \\ \mathrm{i} \omega \chi_{m} \hat{p}(\boldsymbol{x}, \omega)=-\operatorname{div} \hat{\boldsymbol{v}}(\boldsymbol{x}, \omega) & \left(\left|x_{1}\right| \geq a / 2, x_{2} \in \mathbb{R}\right) \\ \llbracket \hat{p} \rrbracket_{a}=B_{1}\left\langle\partial_{1} \hat{p}\right\rangle_{a}+B_{2}\left\langle\partial_{2} \hat{p}\right\rangle_{a} & \left(x_{2} \in \mathbb{R}\right) \\ \llbracket \hat{v}_{1} \rrbracket_{a}=C_{11}\left\langle\partial_{1} \hat{v}_{1}\right\rangle_{a}+C_{12}\left\langle\partial_{2} \hat{v}_{1}\right\rangle_{a}+C_{22}\left\langle\partial_{2} \hat{v}_{2}\right\rangle_{a}+h \mathcal{D}(\omega)\langle\operatorname{div} \hat{\boldsymbol{v}}\rangle_{a} & \left(x_{2} \in \mathbb{R}\right),\end{cases}
$$

where $\partial_{j} \cdot=\frac{\partial}{\partial x_{j}}$ while

$$
\llbracket f(\boldsymbol{x}) \rrbracket_{a}=f\left(a / 2, x_{2}\right)-f\left(-a / 2, x_{2}\right), \quad\langle f(\boldsymbol{x})\rangle_{a}=\frac{1}{2}\left(f\left(a / 2, x_{2}\right)+f\left(-a / 2, x_{2}\right)\right)
$$

denote the jump and the mean value of a given field $f$ relatively to the enlarged interface, respectively. In addition, the constitutive parameters $B_{1}, B_{2}, C_{11}, C_{12}$ and $C_{22}$ featured in (3) 
depend only on the geometry of the inclusions $\Omega_{i}$ and on the material parameters of the matrix and inclusions. Moreover, the term $\mathcal{D}(\omega)$ is a frequency-dependent parameter that encapsulates the resonant behavior of the inclusions. Remarkably, this term can be expanded as:

$$
\mathcal{D}(\omega)=\alpha_{0}-\sum_{r \geq 1} \alpha_{r}^{2} \frac{\omega^{2}}{\omega^{2}-\omega_{r}^{2}}
$$

where $\left\{\omega_{r}\right\}_{r \geq 1}$ is a set of resonant frequencies associated with real-valued coefficients $\left\{\alpha_{r}\right\}_{r \geq 0}$. Note that the non-resonant effective interface model considered in [12] can formally be recovered by setting $\alpha_{r}=0$ for all $r \geq 0$. Lastly, with the purpose of simulating transient waves using the above meta-interface model, we assume that the sum in (5) can be truncated to a finite number $N_{R}$ of resonances based on the analysis of the frequency content of a given illuminating wave. In the realistic applications studied so far, a number of resonances between 5 and 8 is enough to do time-domain simulations that already go beyond the assumptions made to derive the model.

Remark 1. The derivation of the effective model is beyond the scope of the present study and the model (3) will be considered as such to perform wave propagation simulations hereafter. In particular, the featured constitutive parameters will be chosen independently of a microstructure. Moreover, even if the effective jump conditions are obtained [22] for configurations with zero curvature, they will be employed in Section 4 for $2 D$ configurations involving curved enlarged interfaces as well. In this case, they will be properly expressed using a curvilinear coordinates system.

\subsection{Auxiliary variable-based time-domain formulation}

Owing to the frequency dependence of the term $\mathcal{D}(\omega)$ in $(5)$, then the transposition of the system (3) to the time domain brings up a convolution integral in time. Handling numerically such a non-local term is very costly and would therefore reduce the computational gains associated with the treatment of the microstructured layer through an effective model.

To circumvent this difficulty, the auxiliary variable approach can be used to obtain a set of equations in a local form, see [1] for the application of the latter to dispersive metamaterials in bulk. Accordingly, let define locally on the enlarged interface, the so-called auxiliary variables $\hat{J}_{r}$ and $\hat{G}_{r}$, which are associated with the resonance index $r \in\left\{1, \ldots, N_{R}\right\}$ and satisfy the following equations:

$$
\left\{\begin{array}{l}
\left(\omega^{2}-\omega_{r}^{2}\right) \hat{J}_{r}\left(x_{2}, \omega\right)=\alpha_{r}^{2} \omega^{2}\langle\operatorname{div} \hat{\boldsymbol{v}}(\boldsymbol{x}, \omega)\rangle_{a} \\
\mathrm{i} \omega \hat{J}_{r}\left(x_{2}, \omega\right)=\hat{G}_{r}\left(x_{2}, \omega\right)
\end{array} \quad \text { for } x_{2} \in \mathbb{R} .\right.
$$

Setting $\widetilde{C}_{11}=C_{11}+h \alpha_{0}$ and $\widetilde{C}_{22}=C_{22}+h \alpha_{0}$ while combining (3) and (6) then yields the following system in the time domain for all $t>0$ by a formal application of the inverse Fourier 
transform:

$$
\begin{cases}\partial_{t} \boldsymbol{v}(\boldsymbol{x}, t)=-\frac{1}{\rho_{m}} \boldsymbol{\nabla} p(\boldsymbol{x}, t) & \left(\left|x_{1}\right| \geq a / 2, x_{2} \in \mathbb{R}\right) \\ \partial_{t} p(\boldsymbol{x}, t)=-\frac{1}{\chi_{m}} \operatorname{div} \boldsymbol{v}(\boldsymbol{x}, t) & \left(\left|x_{1}\right| \geq a / 2, x_{2} \in \mathbb{R}\right) \\ \partial_{t} G_{r}\left(x_{2}, t\right)=-\omega_{r}^{2} J_{r}\left(x_{2}, t\right)+\left(c_{m} \alpha_{r}\right)^{2}\langle\Delta \operatorname{div} \boldsymbol{v}(\boldsymbol{x}, t)\rangle_{a} & \left(x_{2} \in \mathbb{R}, r=1, \ldots, N_{R}\right) \\ \partial_{t} J_{r}\left(x_{2}, t\right)=G_{r}\left(x_{2}, t\right) & \left(x_{2} \in \mathbb{R}, r=1, \ldots, N_{R}\right) \\ \llbracket p \rrbracket_{a}=B_{1}\left\langle\partial_{1} p\right\rangle_{a}+B_{2}\left\langle\partial_{2} p\right\rangle_{a} & \left(x_{2} \in \mathbb{R}\right) \\ \llbracket v_{1} \rrbracket_{a}=\widetilde{C}_{11}\left\langle\partial_{1} v_{1}\right\rangle_{a}+C_{12}\left\langle\partial_{2} v_{1}\right\rangle_{a}+\widetilde{C}_{22}\left\langle\partial_{2} v_{2}\right\rangle_{a}-h \sum_{r=1}^{N_{R}} J_{r} & \left(x_{2} \in \mathbb{R}\right) .\end{cases}
$$

Note that, to be consistent with the definition (6), the auxiliary variables $J_{r}$ are not averaged in the last equation of the above system.

Remark 2. The auxiliary variables $\left\{\hat{J}_{r}\right\}$ are defined through (6) for all $\omega \neq \omega_{r}$. Therefore, in order to apply the inverse Fourier transform, care must be taken and a suitable approach relies on the introduction of an artificial damping parameter that is taken to zero once in the time domain. Studying such a limit amounts to investigate the question of the existence of a limiting absorption principle for the system considered, which is however beyond the scope of this work. Reference can be made to, e.g., [5] for the treatment of this question for dispersive media in electromagnetism.

Starting from the original variable set $\boldsymbol{u}=(p, \boldsymbol{v})^{\top}$, the set $\boldsymbol{w}=\left(\left\{J_{r}\right\},\left\{G_{r}\right\}\right)^{\top}$ of auxiliary variables is introduced locally along the enlarged interface to allow the derivation of the system (7). The latter consists of first-order equations in time that are complemented by jump conditions that are local in time. Considering the complete set of variables $\tilde{\boldsymbol{u}}=(\boldsymbol{u}, \boldsymbol{w})^{\top}$ then (7) can be written in a compact form for all $t>0$ as:

$$
\begin{cases}\partial_{t} \boldsymbol{u}+\mathbb{A}_{j} \partial_{j} \boldsymbol{u}=\mathbf{0} & \left(\left|x_{1}\right| \geq a / 2, x_{2} \in \mathbb{R}\right), \\ \partial_{t} \boldsymbol{w}+\mathbb{B}\left\langle\partial_{j}^{2} \partial_{\ell} \boldsymbol{u}\right\rangle_{a}+\mathbb{C} \boldsymbol{w}=\mathbf{0} & \left(x_{2} \in \mathbb{R}\right), \\ \llbracket \boldsymbol{u} \rrbracket_{a}=\mathbb{D}_{j}\left\langle\partial_{j} \boldsymbol{u}\right\rangle_{a}+\mathbb{E} \boldsymbol{w} & \left(x_{2} \in \mathbb{R}\right),\end{cases}
$$

using the Einstein summation convention for repeated indices and where the matrices $\mathbb{A}_{j}, \mathbb{B}$, $\mathbb{C}, \mathbb{D}_{j}$ and $\mathbb{E}$ concatenate the parameters characterizing the resonant meta-interface model considered.

\section{Numerical modeling: detailed implementation in 1D}

\subsection{Preliminaries}

In this section, we describe the numerical implementation of the system (7) in a onedimensional configuration which is representative of the propagation of a plane wave illuminating a straight enlarged interface at normal incidence in $2 \mathrm{D}$. The velocity reduces to the scalar 
field $\boldsymbol{v}(\boldsymbol{x})=v(x) \boldsymbol{e}_{1}$ where $x=\boldsymbol{x} \cdot \boldsymbol{e}_{1}$ with $\left(\boldsymbol{e}_{1}, \boldsymbol{e}_{2}\right)$ being the canonical basis of $\mathbb{R}^{2}$. In this setting, the system (7) is recast for all $t>0$ as:

$$
\begin{cases}\partial_{t} v(x, t)=-\frac{1}{\rho_{m}} \partial_{x} p(x, t) & (|x| \geq a / 2), \\ \partial_{t} p(x, t)=-\frac{1}{\chi_{m}} \partial_{x} v(x, t) & (|x| \geq a / 2), \\ \partial_{t} G_{r}(t)=-\omega_{r}^{2} J_{r}(t)+\left(c_{m} \alpha_{r}\right)^{2}\left\langle\partial_{x}^{3} v(x, t)\right\rangle_{a} & \left(r=1, \ldots, N_{R}\right), \\ \partial_{t} J_{r}(t)=G_{r}(t) & \left(r=1, \ldots, N_{R}\right), \\ \llbracket p(x, t) \rrbracket_{a}=B_{1}\left\langle\partial_{x} p(x, t)\right\rangle_{a}, & \\ \llbracket v(x, t) \rrbracket_{a}=\widetilde{C}_{11}\left\langle\partial_{x} v(x, t)\right\rangle_{a}-h \sum_{r=1}^{N_{R}} J_{r}(t) . & \end{cases}
$$

According to the condensed form (8), we introduce the matrix

$$
\mathbb{A}=\left(\begin{array}{cc}
0 & 1 / \chi_{m} \\
1 / \rho_{m} & 0
\end{array}\right)
$$

so that summing up (9a) and (9b) yields

$$
\partial_{t} \boldsymbol{u}+\mathbb{A} \partial_{x} \boldsymbol{u}=\mathbf{0} \quad(|x| \geq a / 2) .
$$

\section{2. $A D E R-K$ scheme}

The solution $\boldsymbol{u}$ is discretized with a mesh size $\Delta x$ and a time step $\Delta t$. We denote as $\boldsymbol{u}_{i}^{n}$ the approximation of $\boldsymbol{u}$ at the point $x_{i}=i \Delta x$ and time $t_{n}=n \Delta t$. The explicit finite-difference ADER- $K$ scheme [25, 17], with $K$ an even integer, is used to solve numerically (11). When applied to an equation such as (11) in a homogeneous domain, this scheme is of order $K$ in both space and time for sufficiently smooth initial data and, for $K=4$, it is stable under the CFL condition $\Delta t \leq \Delta x / c_{m}$. Its time-marching scheme writes:

$$
\boldsymbol{u}_{i}^{n+1}=\boldsymbol{u}_{i}^{n}-\sum_{s=-K / 2}^{+K / 2} \sum_{m=1}^{K} \nu_{K, m, s}\left(\frac{\Delta t}{\Delta x} \mathbb{A}\right)^{m} \boldsymbol{u}_{i+s}^{n},
$$

where the $\nu_{K, m, s}$ are a set of coefficients such that, if the solution $\boldsymbol{u}$ is of class $C^{K}$, then its $m$-th order derivative can be approximated as [11]:

$$
\partial_{x}^{m} \boldsymbol{u}\left(x_{j}, t_{n}\right)=\frac{(-1)^{m+1}}{\Delta x^{m}} m ! \sum_{s=-K / 2}^{K / 2} \nu_{K, m, s} \boldsymbol{u}\left(x_{j+s}, t_{n}\right)+\mathcal{O}\left(\Delta x^{K+1}\right), \quad m=1, \ldots, K .
$$

Inserting Taylor expansions for $\boldsymbol{u}\left(x_{j+s}, t_{n}\right)$ at $\left(x_{j}, t_{n}\right)$ and up to the order $K$ in the previous expression leads to the following relations that are satisfied by the coefficients $\nu_{K, m, s}$ for $0 \leq$ $k \leq K$ and $1 \leq m \leq K$ :

$$
\sum_{s=-K / 2}^{K / 2} \nu_{K, m, s} s^{k}= \begin{cases}(-1)^{m+1} & \text { if } k=m \\ 0 & \text { else. }\end{cases}
$$

For now on, we choose the ADER scheme with $K=4$ which we consider to allow a good compromise between accuracy and ease of implementation. 


\subsection{Numerical scheme at the interfaces}

Considering the time-marching scheme (12), two types of points can be distinguished, see Figure 2: (i) regular points that are the grid nodes for which the stencil does not intersect the enlarged interface, and (ii) irregular points that are the nodes whose stencil includes at least one node within the enlarged interface where the solution is actually not defined. Such grid nodes $x_{i}$ lying within the enlarged interface are referred to as phantom points. For time-marching at the irregular points, the scheme (12) is modified: phantom values $\boldsymbol{u}^{*}$ have to be defined and used at the phantom points $x_{i}$ within the enlarged interface while standard numerical values can be used otherwise. In the framework of the Explicit Simplified Interface Method (ESIM), these phantom values are defined as smooth extrapolations of the solution at the phantom points from the values of the solution at the physical points $\pm a / 2$. In the case of the ADER-4 scheme, they are given by

$$
\begin{cases}\boldsymbol{u}^{*}\left(x_{i}, t_{n}\right)=\sum_{m=0}^{q} \frac{1}{m !}\left(x_{i}+\frac{a}{2}\right)^{m} \partial_{x}^{m} \boldsymbol{u}\left(-\frac{a}{2}, t_{n}\right) & \text { for } i=I_{L}+1, I_{L}+2, \\ \boldsymbol{u}^{*}\left(x_{i}, t_{n}\right)=\sum_{m=0}^{q} \frac{1}{m !}\left(x_{i}-\frac{a}{2}\right)^{m} \partial_{x}^{m} \boldsymbol{u}\left(\frac{a}{2}, t_{n}\right) \quad \text { for } i=I_{R}-1, I_{R}-2,\end{cases}
$$

where $I_{L}$ and $I_{R}$ are the indices of the grid nodes that are the closest to the enlarged interface on each side and $q>1$ is a user-chosen parameter that controles the accuracy of the approximation.

Remark 3. Note that in the definition (15) of the phantom values, the point $x_{i}$ is a grid node that is situated in the enlarged interface while $\pm a / 2$ is a physical point that may not coincide with a grid node. This is the whole point of the ESIM, which allows to implement jump conditions at interfaces whose geometry may be independent of the computational grid of the finite-difference scheme considered.

This methodology has been implemented in the case of non-resonant effective interface models in [12]. It is extended here to the case of resonant models, which requires substantial modifications due to use of auxiliary variables. In the sections 3.4 and 3.5 , it is shown how the computation of the phantom values through (15) rely both on the use of the jump conditions (9e-9f) and on Taylor expansions on both sides of the enlarged interface.
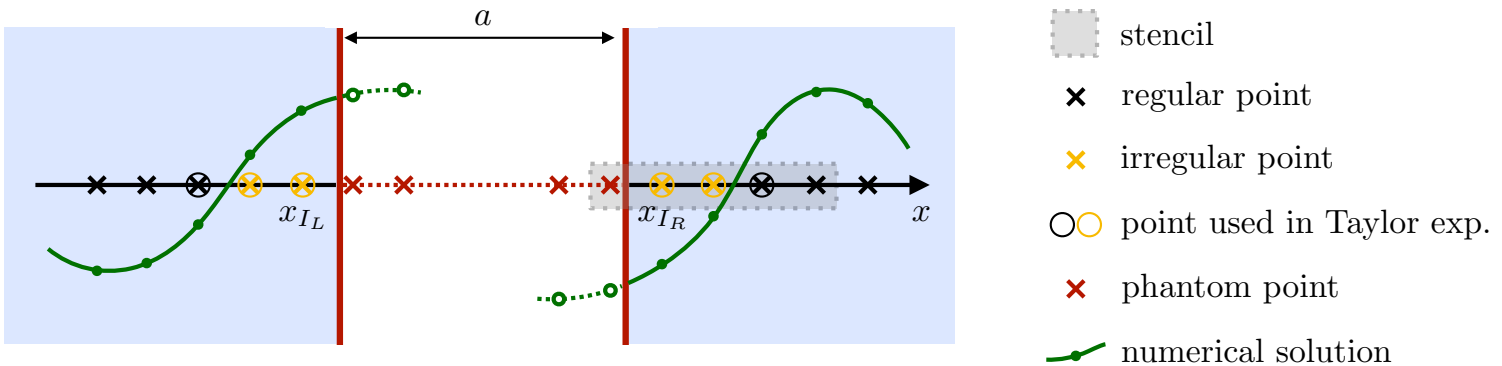

Figure 2: Nodes surrounding the enlarged interface for the ADER-4 scheme. 


\subsection{High-order jump conditions}

The phantom values $\boldsymbol{u}^{*}$ in (15) are expressed in terms of the spatial derivatives $\partial_{x}^{m} \boldsymbol{u}$ at $\pm a / 2$. To compute the latter, one is required to derive $q$-th order jump conditions relating the traces of the spatial derivatives of the solution, up to its $q$-th derivative. Some notations are introduced: the vectors $\boldsymbol{U}_{+}^{q}\left(t_{n}\right)$ and $\boldsymbol{U}_{-}^{q}\left(t_{n}\right)$ concatenate $q_{U}=(2 q+2)$ unknowns which are the traces of the spatial derivatives of the fields $p$ and $v$ up to order $q$ and on each side as

$$
\boldsymbol{U}_{ \pm}^{q}\left(t_{n}\right)=\left(p_{ \pm}\left(t_{n}\right), \partial_{x} p_{ \pm}\left(t_{n}\right), \ldots, \partial_{x}^{q} p_{ \pm}\left(t_{n}\right), v_{ \pm}\left(t_{n}\right), \partial_{x} v_{ \pm}\left(t_{n}\right), \ldots, \partial_{x}^{q} v_{ \pm}\left(t_{n}\right)\right)^{\top} \in \mathbb{R}^{q_{U}},
$$

where $p_{ \pm}\left(t_{n}\right) \equiv p\left( \pm a / 2, t_{n}\right)$ and $v_{ \pm}\left(t_{n}\right) \equiv v\left( \pm a / 2, t_{n}\right)$ and employing the same notation for the spatial derivatives. Index numbering starts at 1 for all the vectors and matrices considered in this paper. Moreover, the vector $\boldsymbol{Z}$ contains the $2 N_{R}$ auxiliary variables at time $t_{n}$, i.e.

$$
\boldsymbol{Z}\left(t_{n}\right)=\left(J_{1}\left(t_{n}\right), \ldots, J_{N_{R}}\left(t_{n}\right), G_{1}\left(t_{n}\right), \ldots, G_{N_{R}}\left(t_{n}\right)\right)^{\top} \in \mathbb{R}^{2 N_{R}} .
$$

To obtain jump conditions at the order $q$, i.e. a relation between the vectors $\boldsymbol{U}_{+}^{q}, \boldsymbol{U}_{-}^{q}$ and $\boldsymbol{Z}$, the zero-th order jump conditions (9e) and (9f) are first differentiated in time and the equations (9a) and (9b) are used to replace time derivatives by spatial derivatives. This yields a relation between the vectors $\boldsymbol{U}_{+}^{1}, \boldsymbol{U}_{-}^{1}$ and $\boldsymbol{Z}$. This process of differentiating in time the jump conditions is iterated up to the chosen value of the parameter $q$ while the equation (9c) is used to expressed the term $\partial_{t} G_{r}$ using $J_{r}$ and $\left\langle\partial_{x}^{3} v\right\rangle_{a}$ when necessary. The $q$-th order jump conditions so obtained are written as:

$$
\mathbb{C}_{+}^{q} \boldsymbol{U}_{+}^{q}\left(t_{n}\right)+\boldsymbol{R}_{+}^{q}\left(t_{n}\right)=\mathbb{C}_{-}^{q} \boldsymbol{U}_{-}^{q}\left(t_{n}\right)+\boldsymbol{R}_{-}^{q}\left(t_{n}\right)+\mathbb{Q}^{q} \boldsymbol{Z}\left(t_{n}\right)
$$

with $\mathbb{C}_{ \pm}^{q}$ being $q_{U} \times q_{U}$ matrices that depend only on the interface parameters and $\mathbb{C}_{+}^{q}$ being invertible in the cases considered hereafter. $\mathbb{Q}^{q}$ is a $q_{U} \times 2 N_{R}$ matrix that depends only on the physical parameters and on the resonant frequencies. The $q_{U^{-}}$element vectors $\boldsymbol{R}_{ \pm}^{q}\left(t_{n}\right)$ contain the $(q+1)$-th order derivatives of $p$ and $v$.

In the ensuing numerical examples, the values $q=3$ and $q=5$ are chosen and it is checked numerically that the corresponding matrices $\mathbb{C}_{+}^{q}$ are invertible. In the case $q=3$ the entries of the above matrices are given below:

$$
\begin{aligned}
& \begin{cases}\mathbb{C}_{ \pm}^{3}[i, i]=1, & \mathbb{C}_{ \pm}^{3}[5,6]=\mp \frac{\widetilde{C}_{11}}{2} \\
\mathbb{C}_{ \pm}^{3}[1,2]=\mp \frac{B_{1}}{2}, & \mathbb{C}_{ \pm}^{3}[6,7]=\mp \frac{B_{1}}{2} \\
\mathbb{C}_{ \pm}^{3}[2,3]=\mp \frac{\widetilde{C}_{11}}{2}, & \mathbb{C}_{ \pm}^{3}[7,8]=\mp \frac{1}{2}\left(\widetilde{C}_{11}-h \sum_{r=1}^{N_{R}} \alpha_{r}^{2}\right), \\
\mathbb{C}_{ \pm}^{3}[3,4]=\mp \frac{B_{1}}{2}, & \mathbb{C}_{ \pm}^{3}[i, j]=0 \text { else. }\end{cases}
\end{aligned}
$$

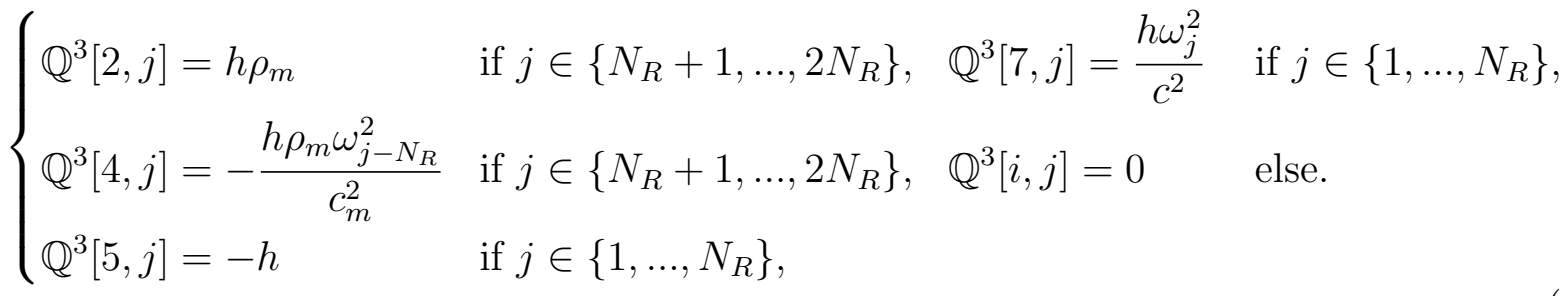

$$
\begin{aligned}
& \left\{\boldsymbol{R}_{ \pm}^{3}[4]=\mp \frac{1}{2}\left(\widetilde{C}_{11}-h \sum_{r=1}^{N_{R}} \alpha_{r}^{2}\right) \partial_{x}^{4} p_{ \pm}, \quad \boldsymbol{R}_{ \pm}^{3}[8]=\mp \frac{B_{1}}{2} \partial_{x}^{4} v_{ \pm}, \quad \boldsymbol{R}_{ \pm}^{3}[i]=0\right. \text { else }
\end{aligned}
$$




\subsection{Computation of the phantom values}

Consider $\mathbb{T}_{i}^{q}( \pm a / 2)$ as the $2 \times q_{U}$ matrices of the polynomial forms of the Taylor expansions at the order $q$ between the grid node with index $i$ and the physical point $\pm a / 2$, which may not coincide with a grid point, see Remark 3, i.e.

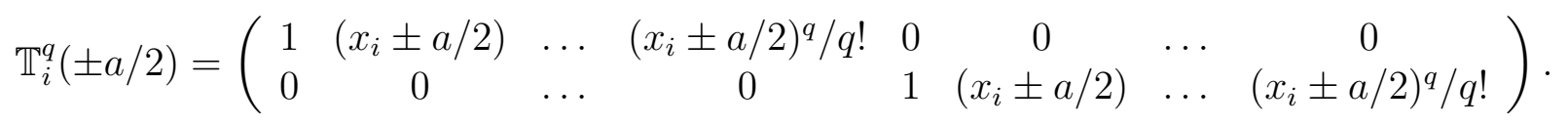

The equation (15) is now recast as

$$
\begin{cases}\boldsymbol{u}^{*}\left(x_{i}, t_{n}\right)=\mathbb{T}_{i}^{q}\left(-\frac{a}{2}\right) \boldsymbol{U}_{-}^{q}\left(t_{n}\right) & \text { for } i=I_{L}+1, I_{L}+2 \\ \boldsymbol{u}^{*}\left(x_{i}, t_{n}\right)=\mathbb{T}_{i}^{q}\left(\frac{a}{2}\right) \boldsymbol{U}_{+}^{q}\left(t_{n}\right) & \text { for } i=I_{R}-1, I_{R}-2 .\end{cases}
$$

The vector $\boldsymbol{U}_{+}^{q}\left(t_{n}\right)$ can be expressed as a function of $\boldsymbol{U}_{-}^{q}\left(t_{n}\right)$ using the $q$-th order jump conditions (18). Now, to determine $\boldsymbol{U}_{-}^{q}\left(t_{n}\right)$ we introduce a user-chosen parameter $q_{T}$ according to which Taylor expansions are written out at the $q_{T}$ nodes $x_{i}=x_{I_{L}-q_{T}+1}, \ldots, x_{I_{L}}$ on the left side of the enlarged interface as

$$
\boldsymbol{u}\left(x_{i}, t_{n}\right)=\mathbb{T}_{i}^{q}\left(-\frac{a}{2}\right) \boldsymbol{U}_{-}^{q}\left(t_{n}\right)+\mathcal{O}\left(\Delta x^{q+1}\right),
$$

and at the $q_{T}$ nodes $x_{i}=x_{I_{R}}, \ldots, x_{I_{R}+q_{T}-1}$ on the right side of the enlarged interface as

$$
\begin{aligned}
\boldsymbol{u}\left(x_{i}, t_{n}\right) & =\mathbb{T}_{i}^{q}\left(\frac{a}{2}\right) \boldsymbol{U}_{+}^{q}\left(t_{n}\right)+\mathcal{O}\left(\Delta x^{q+1}\right) \\
& =\mathbb{T}_{i}^{q}\left(\frac{a}{2}\right)\left(\mathbb{C}_{+}^{q}\right)^{-1}\left[\mathbb{C}_{-}^{q} \boldsymbol{U}_{-}^{q}\left(t_{n}\right)+\mathbb{Q}^{q} \boldsymbol{Z}\left(t_{n}\right)+\boldsymbol{R}_{-}^{q}\left(t_{n}\right)-\boldsymbol{R}_{+}^{q}\left(t_{n}\right)\right]+\mathcal{O}\left(\Delta x^{q+1}\right) .
\end{aligned}
$$

In (24), we made use of (18) and, by an abuse of notations, the term $\mathcal{O}\left(\Delta x^{q+1}\right)$ denotes a vector with entries of the order of $\Delta x^{q+1}$ and whose size may vary from line to line. We introduce the $4 q_{T} \times q_{U}$ matrices $\mathbb{M}^{q}$ and $\mathbb{F}^{q}$ by blocks:

$$
\mathbb{M}^{q}=\left(\begin{array}{c}
\mathbb{T}_{I_{L}-q_{T}+1}^{q}\left(-\frac{a}{2}\right) \\
\vdots \\
\mathbb{T}_{I_{L}}^{q}\left(-\frac{a}{2}\right) \\
\mathbb{T}_{I_{R}}^{q}\left(\frac{a}{2}\right)\left(\mathbb{C}_{+}^{q}\right)^{-1} \mathbb{C}_{-}^{q} \\
\vdots \\
\mathbb{T}_{I_{R}+q_{T}-1}^{q}\left(\frac{a}{2}\right)\left(\mathbb{C}_{+}^{q}\right)^{-1} \mathbb{C}_{-}^{q}
\end{array}\right), \quad \mathbb{F}^{q}=\left(\begin{array}{c}
\mathbf{0} \\
\vdots \\
\mathbf{0} \\
-\mathbb{T}_{I_{R}}^{q}\left(\frac{a}{2}\right) \\
\vdots \\
-\mathbb{T}_{I_{R}+q_{T}-1}^{q}\left(\frac{a}{2}\right)
\end{array}\right)\left(\mathbb{C}_{+}^{q}\right)^{-1}
$$

and the $4 q_{T}$-element vectors $\mathcal{U}\left(t_{n}\right)$ and $\boldsymbol{\Delta}\left(t_{n}\right)$ :

$$
\mathcal{U}\left(t_{n}\right)=\left(\begin{array}{c}
\boldsymbol{u}\left(x_{I_{L}-q_{T}+1}, t_{n}\right) \\
\vdots \\
\boldsymbol{u}\left(x_{I_{L}}, t_{n}\right) \\
\boldsymbol{u}\left(x_{I_{R}}, t_{n}\right) \\
\vdots \\
\boldsymbol{u}\left(x_{I_{R}+q_{T}-1}, t_{n}\right)
\end{array}\right), \quad \boldsymbol{\Delta}\left(t_{n}\right)=-\mathbb{F}^{q}\left(\boldsymbol{R}_{-}^{q}\left(t_{n}\right)-\boldsymbol{R}_{+}^{q}\left(t_{n}\right)\right)
$$


Then, from (23) and (24), one obtains the following system:

$$
\mathbb{M}^{q} \boldsymbol{U}_{-}^{q}\left(t_{n}\right)=\mathcal{U}\left(t_{n}\right)+\mathbb{F}^{q} \mathbb{Q}^{q} \boldsymbol{Z}\left(t_{n}\right)-\boldsymbol{\Delta}\left(t_{n}\right)+\mathcal{O}\left(\Delta x^{q+1}\right)
$$

When the parameter $q_{T}$ is chosen as $4 q_{T}=q_{U}$, then $\mathbb{M}^{q}$ is a square matrix that is formally checked to be invertible. In order to work as often as possible with a square matrix, $q$ is always chosen odd in $1 \mathrm{D}$. If $4 q_{T}>q_{U}$ then $\mathbb{M}^{q}$ is not square and, in such a case, we denote by $\left(\mathbb{M}^{q}\right)^{-1}$ its Moore-Penrose pseudo-inverse. Therefore, using the $q$-th order jump conditions (18), we arrive at the following expressions for the vectors $\boldsymbol{U}_{ \pm}^{q}\left(t_{n}\right)$ :

$$
\boldsymbol{U}_{-}^{q}\left(t_{n}\right)=\left(\mathbb{M}^{q}\right)^{-1}\left(\mathcal{U}\left(t_{n}\right)+\mathbb{F}^{q} \mathbb{Q}^{q} \boldsymbol{Z}\left(t_{n}\right)-\boldsymbol{\Delta}\left(t_{n}\right)+\mathcal{O}\left(\Delta x^{q+1}\right)\right),
$$

and

$$
\begin{aligned}
\boldsymbol{U}_{+}^{q}\left(t_{n}\right) & =\left(\mathbb{C}_{+}^{q}\right)^{-1}\left[\mathbb{C}_{-}^{q}\left(\mathbb{M}^{q}\right)^{-1}\left(\mathcal{U}\left(t_{n}\right)+\mathbb{F}^{q} \mathbb{Q}^{q} \boldsymbol{Z}\left(t_{n}\right)-\boldsymbol{\Delta}\left(t_{n}\right)+\mathcal{O}\left(\Delta x^{q+1}\right)\right)\right. \\
& \left.+\mathbb{Q}^{q} \boldsymbol{Z}\left(t_{n}\right)+\boldsymbol{R}_{-}^{q}\left(t_{n}\right)-\boldsymbol{R}_{+}^{q}\left(t_{n}\right)\right] .
\end{aligned}
$$

Neglecting the terms that do not depend on $\mathcal{U}\left(t_{n}\right)$ or $\boldsymbol{Z}\left(t_{n}\right)$ in (28) and (29), and introducing the $q_{U} \times q_{U}$ matrix $\mathbb{G}^{q}$ as

$$
\mathbb{G}^{q}=\mathbb{C}_{-}^{q}\left(\mathbb{M}^{q}\right)^{-1} \mathbb{F}^{q}+\mathbb{I}_{q_{U}},
$$

then allows us to formulate the numerical approximation $\left(\boldsymbol{U}_{ \pm}^{q}\right)^{n}$ of the vectors $\boldsymbol{U}_{ \pm}^{q}\left(t_{n}\right)$ :

$$
\left(\boldsymbol{U}_{-}^{q}\right)^{n}=\left(\mathbb{M}^{q}\right)^{-1}\left[\mathcal{U}^{n}+\mathbb{F}^{q} \mathbb{Q}^{q} \boldsymbol{Z}^{n}\right]
$$

and

$$
\left(\boldsymbol{U}_{+}^{q}\right)^{n}=\left(\mathbb{C}_{+}^{q}\right)^{-1}\left[\mathbb{C}_{-}^{q}\left(\mathbb{M}^{q}\right)^{-1} \mathcal{U}^{n}+\mathbb{G}^{q} \mathbb{Q}^{q} \boldsymbol{Z}^{n}\right]
$$

Due to (22), we finally arrive at the approximation below for the phantom values.

Approximation 1. The numerical approximations $\left(\boldsymbol{u}^{*}\right)_{i}^{n}$ of the phantom values $\boldsymbol{u}^{*}\left(x_{i}, t_{n}\right)$ are computed using the formulae

$$
\begin{cases}\left(\boldsymbol{u}^{*}\right)_{i}^{n}=\mathbb{T}_{i}^{q}\left(-\frac{a}{2}\right)\left(\mathbb{M}^{q}\right)^{-1}\left[\mathcal{U}^{n}+\mathbb{F}^{q} \mathbb{Q}^{q} \boldsymbol{Z}^{n}\right] & \text { for } i=I_{L}+1, I_{L}+2, \\ \left(\boldsymbol{u}^{*}\right)_{i}^{n}=\mathbb{T}_{i}^{q}\left(\frac{a}{2}\right)\left(\mathbb{C}_{+}^{q}\right)^{-1}\left[\mathbb{C}_{-}^{q}\left(\mathbb{M}^{q}\right)^{-1} \mathcal{U}^{n}+\mathbb{G}^{q} \mathbb{Q}^{q} \boldsymbol{Z}^{n}\right] & \text { for } i=I_{R}-2, I_{R}-1,\end{cases}
$$

in terms of the numerical approximations $\mathcal{U}^{n}, \boldsymbol{Z}^{n}$ of $\mathcal{U}\left(t_{n}\right), \boldsymbol{Z}\left(t_{n}\right)$, respectively.

The quality of this approximation is quantified in Property 1 through a local error analysis. In practice, the matrices $\mathbb{T}_{i}^{q}\left( \pm \frac{a}{2}\right), \mathbb{C}_{ \pm}^{q}, \mathbb{F}^{q}, \mathbb{G}^{q}, \mathbb{Q}^{q}$ and $\left(\mathbb{M}^{q}\right)^{-1}$ featured in (33) are computed in a pre-processing step once for all.

The phantom values (33) are used in the ADER- $K$ scheme (12) at irregular points. For example, the time-stepping at the irregular point $x_{I_{L}}$ on the left side of the enlarged interface is recast as

$$
\begin{aligned}
\frac{\boldsymbol{u}_{I_{L}}^{n+1}-\boldsymbol{u}_{I_{L}}^{n}}{\Delta t} & +\frac{1}{\Delta t} \sum_{s=-K / 2}^{0} \sum_{m=1}^{K} \nu_{K, m, s}\left(\mathbb{A} \frac{\Delta t}{\Delta x}\right)^{m} \boldsymbol{u}_{I_{L}+s}^{n} \\
& +\frac{1}{\Delta t} \sum_{s=1}^{K / 2} \sum_{m=1}^{K} \nu_{K, m, s}\left(\mathbb{A} \frac{\Delta t}{\Delta x}\right)^{m} \boldsymbol{u}_{I_{L}+s}^{* n}=\mathbf{0},
\end{aligned}
$$


which can be written as

$$
\boldsymbol{u}_{I_{L}}^{n+1}=\mathcal{H}_{L}\left(\boldsymbol{u}^{n}, \boldsymbol{Z}^{n}\right)
$$

with $\boldsymbol{u}^{n}=\left\{\boldsymbol{u}_{i}^{n}\right\}_{i}$ being arranged as a vector and $\mathcal{H}_{L}$ defined by

$$
\begin{aligned}
\mathcal{H}_{L}\left(\boldsymbol{u}^{n}, \boldsymbol{Z}^{n}\right)=\boldsymbol{u}_{I_{L}}^{n} & -\sum_{s=-K / 2}^{0} \sum_{m=1}^{K} \nu_{K, m, s}\left(\mathbb{A} \frac{\Delta t}{\Delta x}\right)^{m} \boldsymbol{u}_{I_{L}+s}^{n} \\
& -\sum_{s=1}^{K / 2} \sum_{m=1}^{K} \nu_{K, m, s}\left(\mathbb{A} \frac{\Delta t}{\Delta x}\right)^{m} \mathbb{T}_{I_{L}+s}^{q}\left(-\frac{a}{2}\right)\left(\mathbb{M}^{q}\right)^{-1} \mathcal{U}^{n} \\
& -\sum_{s=1}^{K / 2} \sum_{m=1}^{K} \nu_{K, m, s}\left(\mathbb{A} \frac{\Delta t}{\Delta x}\right)^{m} \mathbb{T}_{I_{L}+s}^{q}\left(-\frac{a}{2}\right)\left(\mathbb{M}^{q}\right)^{-1} \mathbb{F}^{q} \mathbb{Q}^{q} \boldsymbol{Z}^{n}
\end{aligned}
$$

The numerical approximation $\mathcal{U}^{n}$ of $\mathcal{U}\left(t_{n}\right)$ defined in (26) can be expressed as a function of $\boldsymbol{u}^{n}$. On the right side, we take the example of the irregular point $I_{R}$, where the time-stepping can be written:

$$
\boldsymbol{u}_{I_{R}}^{n+1}=\mathcal{H}_{R}\left(\boldsymbol{u}^{n}, \boldsymbol{Z}^{n}\right)
$$

with $\mathcal{H}_{R}$ defined by

$$
\begin{aligned}
\mathcal{H}_{R}\left(\boldsymbol{u}^{n}, \boldsymbol{Z}^{n}\right)=\boldsymbol{u}_{I_{R}}^{n} & -\sum_{s=0}^{K / 2} \sum_{m=1}^{K} \nu_{K, m, s}\left(\mathbb{A} \frac{\Delta t}{\Delta x}\right)^{m} \boldsymbol{u}_{I_{R}+s}^{n} \\
& -\sum_{s=-K / 2}^{-1} \sum_{m=1}^{K} \nu_{K, m, s}\left(\mathbb{A} \frac{\Delta t}{\Delta x}\right)^{m} \mathbb{T}_{I_{R}+s}^{q}\left(\frac{a}{2}\right)\left(\mathbb{C}_{+}^{q}\right)^{-1} \mathbb{C}_{-}^{q}\left(\mathbb{M}^{q}\right)^{-1} \mathcal{U}^{n} \\
& -\sum_{s=-K / 2}^{-1} \sum_{m=1}^{K} \nu_{K, m, s}\left(\mathbb{A} \frac{\Delta t}{\Delta x}\right)^{m} \mathbb{T}_{I_{R}+s}^{q}\left(\frac{a}{2}\right)\left(\mathbb{C}_{+}^{q}\right)^{-1} \mathbb{G}^{q} \mathbb{Q}^{q} \boldsymbol{Z}^{n}
\end{aligned}
$$

Remark 4. In this article, the computation of the phantom values has been discribed for the ADER-4 scheme for the sake of simplicity. Yet, the only difference between the different ADER$K$ schemes is the number of irregular points and phantom points to be considered. Therefore, $\mathcal{H}_{L}$ and $\mathcal{H}_{R}$ at $x_{I_{L}}$ and $x_{I_{R}}$, which are always irregular points, are introduced above for an arbitrary ADER-K scheme, with $K$ being an even integer.

\subsection{Computation of the auxiliary variables}

The computation of the phantom values $\boldsymbol{u}^{*}$ through Approximation 1 requires to determine an approximation $\boldsymbol{Z}^{n}$ of the vector $\boldsymbol{Z}\left(t_{n}\right)$ of auxiliary variables (17) at each time step. Assuming zero initial conditions for $J_{r}$ and $G_{r}$, which is justified in the case where the enlarged interface is illuminated by a remote source, then integrating $(9 \mathrm{c}-9 \mathrm{~d})$ yields

$$
\left\{\begin{array}{l}
J_{r}\left(t_{n}\right)=\Lambda_{r}^{1}\left(t_{n}\right) \cos \left(\omega_{r} t_{n}\right)-\Lambda_{r}^{2}\left(t_{n}\right) \sin \left(\omega_{r} t_{n}\right), \\
G_{r}\left(t_{n}\right)=-\omega_{r}\left(\Lambda_{r}^{2}\left(t_{n}\right) \cos \left(\omega_{r} t_{n}\right)+\Lambda_{r}^{1}\left(t_{n}\right) \sin \left(\omega_{r} t_{n}\right)\right),
\end{array}\right.
$$

where, owing to (9a-9b), one has for $i=1,2$ :

$$
\Lambda_{r}^{i}\left(t_{n}\right)=\frac{\alpha_{r}^{2}}{\omega_{r} \rho_{m}} \int_{0}^{t_{n}} \ell_{r}^{i}(\tau) \mathrm{d} \tau \quad \text { with } \quad\left\{\begin{array}{l}
\ell_{r}^{1}(\tau)=\partial_{t}\left\langle\partial_{x}^{2} p\right\rangle_{a}(\tau) \sin \left(\omega_{r} \tau\right) \\
\ell_{r}^{2}(\tau)=\partial_{t}\left\langle\partial_{x}^{2} p\right\rangle_{a}(\tau) \cos \left(\omega_{r} \tau\right)
\end{array}\right.
$$


In practice, the functions $\Lambda_{r}^{i}$ in (40) are computed iteratively as

$$
\left\{\begin{array}{l}
\Lambda_{r}^{i}\left(t_{0}\right)=0 \\
\Lambda_{r}^{i}\left(t_{n+1}\right)=\Lambda_{r}^{i}\left(t_{n}\right)+\frac{\alpha_{r}^{2}}{\omega_{r} \rho_{m}} \int_{t_{n}}^{t_{n+1}} \ell_{r}^{i}(\tau) \mathrm{d} \tau
\end{array}\right.
$$

where $t_{0}=0$ and the integral being computed using the extrapolative Newton-Cotes formulas [27], i.e.

$$
\int_{t_{n}}^{t_{n+1}} \ell_{r}^{i}(\tau) \mathrm{d} \tau=\Delta t \sum_{m=0}^{q_{I}-2} \gamma_{m} \ell_{r}^{i}\left(t_{n-m}\right)+\mathcal{O}\left(\Delta t^{q_{I}}\right) .
$$

The values of the parameters $\gamma_{m}$ for $q_{I}=2, \ldots, 5$ are reported in Table 1 .

\begin{tabular}{|c||c|c|c|c|}
\hline$q_{I}$ & 2 & 3 & 4 & 5 \\
\hline \hline$\gamma_{0}$ & 1 & $3 / 2$ & $23 / 12$ & $55 / 24$ \\
\hline$\gamma_{1}$ & & $-1 / 2$ & $-16 / 12$ & $-59 / 24$ \\
\hline$\gamma_{2}$ & & & $5 / 12$ & $37 / 24$ \\
\hline$\gamma_{3}$ & & & & $-9 / 24$ \\
\hline
\end{tabular}

Table 1: Numerical integration by the Newton-Cotes formulas: values of the parameter $\gamma_{m}$ featured in (42).

Moreover, the computation of the terms $\ell_{r}^{i}\left(t_{n-m}\right)$ defined through in (40) and used in (42) requires to approximate the temporal derivative $\partial_{t}\left\langle\partial_{x}^{2} p\right\rangle_{a}\left(t_{n-m}\right)$, which is achieved using the following finite-difference approximation:

$$
\partial_{t}\left\langle\partial_{x}^{2} p\right\rangle_{a}\left(t_{n-m}\right)=\frac{1}{\Delta t} \sum_{w=0}^{q_{D}} \beta_{w}\left\langle\partial_{x}^{2} p\right\rangle_{a}\left(t_{n-m-w}\right)+\mathcal{O}\left(\Delta t^{q_{D}}\right)
$$

The values of $\beta_{w}$ for $q_{D}=1, \ldots, 4$ are listed in Table 2 .

\begin{tabular}{|c||c|c|c|c|}
\hline$q_{D}$ & 1 & 2 & 3 & 4 \\
\hline \hline$\beta_{0}$ & 1 & $3 / 2$ & $11 / 6$ & $75 / 36$ \\
\hline$\beta_{1}$ & -1 & $-4 / 2$ & $-18 / 6$ & $-144 / 36$ \\
\hline$\beta_{2}$ & & $1 / 2$ & $9 / 6$ & $108 / 36$ \\
\hline$\beta_{3}$ & & & $-2 / 6$ & $-48 / 36$ \\
\hline$\beta_{4}$ & & & & $9 / 36$ \\
\hline
\end{tabular}

Table 2: Numerical derivation: values of the parameter $\beta_{w}$ featured in (43).

Remark 5. Owing to (9c), the term $\left\langle\partial_{x}^{3} v\right\rangle_{a}$ can be used in (40) instead of the quantity $\partial_{t}\left\langle\partial_{x}^{2} p\right\rangle_{a}$ with mixed derivatives in time and space. However, in practice, numerical instabilities have been observed in 2D when the third-order spatial derivative is used, contrary to the mixed approach described here. Such instabilities were not observed in $1 D$ but we adopt this mixed approach for the $1 D$ case as well for consistency. 
By inserting (42) and (43) in (41), while keeping track of the approximation order, we get the following expression for $\Lambda_{r}^{i}$ :

$$
\left\{\begin{array}{l}
\Lambda_{r}^{i}\left(t_{0}\right)=0, \\
\Lambda_{r}^{i}\left(t_{n+1}\right)=\Lambda_{r}^{i}\left(t_{n}\right)+\frac{\alpha_{r}^{2}}{\omega_{r} \rho_{m}} \sum_{m=0}^{q_{I}-2} \gamma_{m} \sum_{w=0}^{q_{D}} \beta_{w}\left\langle\partial_{x}^{2} p\right\rangle_{a}\left(t_{n-m-w}\right)\left(\kappa_{r}^{i}\right)^{n-m}+\mathcal{O}\left(\Delta t^{\min \left(\mathrm{q}_{\mathrm{D}}+1, \mathrm{q}_{\mathrm{I}}\right)}\right)
\end{array}\right.
$$

with $\left(\kappa_{r}^{1}\right)^{s}=\sin \left(\omega_{r} t_{s}\right)$ and $\left(\kappa_{r}^{2}\right)^{s}=\cos \left(\omega_{r} t_{s}\right)$. For $m=0, \ldots, q_{I}-2$ and $w=0, \ldots, q_{D}$, we know from (16) that

$$
\left\langle\partial_{x}^{2} p\right\rangle_{a}\left(t_{n-m-w}\right)=\frac{1}{2}\left(\boldsymbol{U}_{-}^{q}\left(t_{n-m-w}\right)+\boldsymbol{U}_{+}^{q}\left(t_{n-m-w}\right)\right)[3],
$$

where $\boldsymbol{U}[3]$ stands for the third term of the vector $\boldsymbol{U}$. Using the numerical approximations (31) and (32), we obtain the approximation of $\left\langle\partial_{x}^{2} p\right\rangle_{a}\left(t_{n-m-w}\right)$ :

$$
\left\langle\partial_{x}^{2} p\right\rangle_{a}^{n-m-w}=\frac{1}{2}\left[\left(\mathbb{I}_{q_{U}}+\left(\mathbb{C}_{+}^{q}\right)^{-1} \mathbb{C}_{-}^{q}\right)\left(\mathbb{M}^{q}\right)^{-1} \mathcal{U}^{n-m-w}+\left(\left(\mathbb{M}^{q}\right)^{-1} \mathbb{F}^{q}+\left(\mathbb{C}_{+}^{q}\right)^{-1} \mathbb{G}^{q}\right) \mathbb{Q}^{q} \boldsymbol{Z}^{n-m-w}\right][3],
$$

leading to the final approximation below.

Approximation 2. The numerical approximation $\left(\Lambda_{r}^{i}\right)^{n}$ of $\Lambda_{r}^{i}\left(t_{n}\right)$ for $i=1,2$ is computed by the recurrence relation

$$
\left\{\begin{array}{l}
\left(\Lambda_{r}^{i}\right)^{0}=0 \\
\left(\Lambda_{r}^{i}\right)^{n+1}=\left(\Lambda_{r}^{i}\right)^{n}+\frac{\alpha_{r}^{2}}{\omega_{r} \rho_{m}} \sum_{m=0}^{q_{I}-2} \gamma_{m} \sum_{w=0}^{q_{D}} \beta_{w}\left(\left\langle\partial_{x}^{2} p\right\rangle_{a}\right)^{n-m-w}\left(\kappa_{r}^{i}\right)^{n-m}
\end{array}\right.
$$

with $\left(\left\langle\partial_{x}^{2} p\right\rangle_{a}\right)^{s}$ denoting the numerical approximation (46).

For $i=1,2$, we introduce the function $\mathcal{N}_{r}^{i}$ defined by

$$
\begin{aligned}
& \mathcal{N}_{r}^{i}\left(\left(\Lambda_{r}^{i}\right)^{n}, \boldsymbol{u}^{n}, \ldots, \boldsymbol{u}^{n-m-w}, \boldsymbol{Z}^{n}, \ldots, \boldsymbol{Z}^{n-m-w}, t_{n}\right) \\
& =\left(\Lambda_{r}^{i}\right)^{n}+\frac{\alpha_{r}^{2}}{2 \omega_{r} \rho_{m}} \sum_{m=0}^{q_{I}-2} \gamma_{m} \sum_{w=0}^{q_{D}} \beta_{w}\left[\left(\mathbb{I}_{q_{U}}+\left(\mathbb{C}_{+}^{q}\right)^{-1} \mathbb{C}_{-}^{q}\right)\left(\mathbb{M}^{q}\right)^{-1} \mathcal{U}^{n-m-w}\right][3]\left(\kappa_{r}^{i}\right)^{n-m} \\
& +\frac{\alpha_{r}^{2}}{2 \omega_{r} \rho_{m}} \sum_{m=0}^{q_{I}-2} \gamma_{m} \sum_{w=0}^{q_{D}} \beta_{w}\left[\left(\left(\mathbb{M}^{q}\right)^{-1} \mathbb{F}^{q}+\left(\mathbb{C}_{+}^{q}\right)^{-1} \mathbb{G}^{q}\right) \mathbb{Q}^{q} \boldsymbol{Z}^{n-m-w}\right][3]\left(\kappa_{r}^{i}\right)^{n-m},
\end{aligned}
$$

which allows to write that

$$
\left(\Lambda_{r}^{i}\right)^{n+1}=\mathcal{N}_{r}^{i}\left(\left(\Lambda_{r}^{i}\right)^{n}, \boldsymbol{u}^{n}, \ldots, \boldsymbol{u}^{n-m-w}, \boldsymbol{Z}^{n}, \ldots, \boldsymbol{Z}^{n-m-w}, t_{n}\right) .
$$

We now introduce the $2 N_{R} \times 2 N_{R}$ matrix $\mathbb{S}\left(t_{n}\right)$ as follows:

$$
\text { for } j \in\left\{1, \ldots, N_{R}\right\}\left\{\begin{array}{l}
\mathbb{S}[j, j]=\cos \left(\omega_{j} t_{n}\right), \\
\mathbb{S}\left[j, j+N_{R}\right]=-\sin \left(\omega_{j} t_{n}\right), \\
\mathbb{S}\left[j+N_{R}, j\right]=-\omega_{j} \sin \left(\omega_{j} t_{n}\right), \\
\mathbb{S}\left[j+N_{R}, j+N_{R}\right]=-\omega_{j} \cos \left(\omega_{j} t_{n}\right), \\
\mathbb{S}[i, j]=0 \quad \text { else. }
\end{array}\right.
$$

so that, owing to (39), we have the following approximation. 
Approximation 3. The numerical approximation $\boldsymbol{Z}^{n}=\left(\left\{J_{r}^{n}\right\}_{r},\left\{G_{r}^{n}\right\}_{r}\right)$ of the auxiliary variables vector $\boldsymbol{Z}\left(t_{n}\right)=\left(\left\{J_{r}\left(t_{n}\right)\right\}_{r},\left\{G_{r}\left(t_{n}\right)\right\}_{r}\right)$ at the time step $t_{n}$ writes:

$$
Z^{n}=\mathbb{S}\left(t_{n}\right) \Lambda^{n}
$$

with $\Lambda^{n}=\left(\left\{\left(\Lambda_{r}^{1}\right)^{n}\right\}_{r},\left\{\left(\Lambda_{r}^{2}\right)^{n}\right\}_{r}\right)$ being arranged as a vector.

In practice, owing to the formula (47), the computation of the auxiliary variables $J_{r}^{n}, G_{r}^{n}$ at a given time step $t_{n}$ only requires the knowledge of $\left(\Lambda_{r}^{1}\right)^{n-1},\left(\Lambda_{r}^{2}\right)^{n-1}$ and $\left(\left\langle\partial_{x}^{2} p\right\rangle_{a}\right)^{n-1-m-w}$ for $m=0, \ldots,\left(q_{I}-2\right)$ and $w=0, \ldots, q_{D}$. These quantities constitute a set of memory variables, which we regroup in the following vector

$$
\Psi^{n}=\left(\left(\Lambda_{r}^{1}\right)^{n-1},\left(\Lambda_{r}^{2}\right)^{n-1},\left\{\left(\left\langle\partial_{x}^{2} p\right\rangle_{a}\right)^{n-1-m-w}\right\}_{m, w}\right)^{\top}
$$

of size $\left(q_{D}+1\right) \times\left(q_{I}-1\right)+2 N_{R}$. The vector $\boldsymbol{\Psi}^{n}$ is stored and updated during the entire simulation. For a given phantom point, once $\boldsymbol{Z}^{n}$ is computed, the resonant case only requires an additional matrix vector product with a matrix computed in pre-processing. Therefore, in terms of computational time, it is negligible compared to the cost of the numerical scheme.

\subsection{Summary of the algorithm}

\subsection{Numerical analysis}

To the Authors' knowledge, there is no theoretical result available on the numerical stability of the ESIM. In the non-resonant case, no stability issue has been observed on a large number of simulations that involved interfaces with various constitutive parameters and positions within the finite-difference grid [12]. In the resonant case considered in the present study, the stability is observed in practice to depend on the order of integration $q_{I}$ in (42), on the order of derivation $q_{D}$ in (43) on and the number $q_{T}$ of grid nodes considered for the Taylor expansions (23-24). In practice, given $\left(q_{D}, q_{I}\right)$, the stability is observed on numerical experiments for the minimal values of $q_{T}$ that are reported in Table 3 . The case $\left(q_{D}, q_{I}\right)=(4,5)$ is not reported because $q_{T}$ being too large, the use of the pseudo-inverse of the associated matrix $\mathbb{M}^{q}$ yields unacceptable numerical errors. When $q_{T}$ is chosen according to Table 3 , then the CFL condition $\Delta t \leq \Delta x / c_{m}$ of the ADER-4 scheme in a homogeneous domain seems to be the critical threshold for stability here as well.

\begin{tabular}{|ll||l|l|l|l|}
\hline \multicolumn{1}{|c||}{$q_{D}$} & 2 & 3 & 4 & 5 \\
\hline \hline 1 & $q_{U} / 4$ & $q_{U} / 4$ & $q_{U} / 4$ & $q_{U} / 4$ \\
\hline 2 & $q_{U} / 4$ & $q_{U} / 4+1$ & $q_{U} / 4+1$ & $q_{U} / 4+1$ \\
\hline 3 & $q_{U} / 4$ & $q_{U} / 4+1$ & $q_{U} / 4+2$ & $q_{U} / 4+2$ \\
\hline 4 & $q_{U} / 4$ & $q_{U} / 4+1$ & $q_{U} / 4+2$ & \\
\hline
\end{tabular}

Table 3: Minimal value of the Taylor expansion parameter $q_{T}$ for which the scheme is observed to be stable in the numerical experiments considered, given $\left(q_{D}, q_{I}\right)$ and with $q_{U}=(2 q+2)$ as in $(16)$ for $q \in\{3,5\}$.

In this context where no stability result is available then global error estimates cannot be derived for the proposed scheme. Rather, we focus here on a local error analysis. We assume that the numerical solution is the exact continuous one at time $t_{n}$ and evaluate the error 
Algorithm 1 Time-marching scheme with auxiliary variables and phantom values

I. Pre-processing:

1. Detection of the irregular points surrounding the enlarged interface.

2. Computation of the matrices $\left(\mathbb{M}^{q}\right)^{-1}, \mathbb{C}_{-}^{q},\left(\mathbb{C}_{+}^{q}\right)^{-1}, \mathbb{F}^{q}, \mathbb{G}^{q}$ and $\mathbb{Q}^{q}$.

3. Computation of $\mathbb{T}_{i}^{q}\left(-\frac{a}{2}\right)\left(\mathbb{M}^{q}\right)^{-1}$ and $\mathbb{T}_{i}^{q}\left(-\frac{a}{2}\right)\left(\mathbb{M}^{q}\right)^{-1} \mathbb{F}^{q} \mathbb{Q}^{q}$ for phantom values at the left side of the enlarged interface.

4. Computation of $\mathbb{T}_{i}^{q}\left(\frac{a}{2}\right)\left(\mathbb{C}_{+}^{q}\right)^{-1} \mathbb{C}_{-}^{q}\left(\mathbb{M}^{q}\right)^{-1}$ and $\mathbb{T}_{i}^{q}\left(\frac{a}{2}\right)\left(\mathbb{C}_{+}^{q}\right)^{-1} \mathbb{G}^{q} \mathbb{Q}^{q}$ for phantom values at the right side of the enlarged interface.

5. Computation of $\left(\mathbb{I}_{q_{U}}+\left(\mathbb{C}_{+}^{q}\right)^{-1} \mathbb{C}_{-}^{q}\right)\left(\mathbb{M}^{q}\right)^{-1}$ and $\left(\left(\mathbb{M}^{q}\right)^{-1} \mathbb{F}^{q}+\left(\mathbb{C}_{+}^{q}\right)^{-1} \mathbb{G}^{q}\right) \mathbb{Q}^{q}$.

II. Initialization: set the solution $\boldsymbol{u}_{i}^{0}$ at $t_{0}=0$ while $\boldsymbol{\Psi}^{0}=\mathbf{0}$ along the enlarged interface.

III. Iterate in time $n \geq 0$ :

1. Computation of the vector $\boldsymbol{\Lambda}^{n}$ from $\Psi^{n}$ using Approximation 2.

2. Computation of $\mathbb{S}\left(t_{n}\right)$ and then of the auxiliary variables vector $\boldsymbol{Z}^{n}$ from $\boldsymbol{\Lambda}^{n}$ using Approximation 3.

3. Computation of $\mathcal{U}^{n}$ in (26) from $\boldsymbol{u}_{i}^{n}$.

4. Computation of the phantom values $\left(\boldsymbol{u}^{*}\right)_{i}^{n}$ using Approximation 1.

5. Time-marching using (12) to compute the solution $\boldsymbol{u}_{i}^{n+1}$ for all $i$ with the phantom values being used where necessary.

6. Computation of $\left\langle\partial_{x}^{2} p\right\rangle_{a}^{n}$ using (46) and update of the memory variables vector $\Psi^{n+1}$ in $(52)$. 
commited by the numerical scheme in one time step. The expressions of the local errors on $\boldsymbol{u}$, $\boldsymbol{\Lambda}$ and $\boldsymbol{Z}$ at time $t_{n}$ are introduced as:

$$
\left\{\begin{aligned}
\mathcal{L}_{0, \boldsymbol{u}}\left(x_{I_{L}}, t_{n}\right) & =\boldsymbol{u}\left(x_{I_{L}}, t_{n+1}\right)-\mathcal{H}_{L}\left(\boldsymbol{u}\left(\cdot, t_{n}\right), \boldsymbol{Z}\left(t_{n}\right)\right) \\
\mathcal{L}_{0, \boldsymbol{u}}\left(x_{I_{R}}, t_{n}\right) & =\boldsymbol{u}\left(x_{I_{R}}, t_{n+1}\right)-\mathcal{H}_{R}\left(\boldsymbol{u}\left(\cdot, t_{n}\right), \boldsymbol{Z}\left(t_{n}\right)\right) \\
\mathcal{L}_{0, \Lambda_{r}^{i}}\left(t_{n}\right) & =\Lambda_{r}^{i}\left(t_{n}\right)-\mathcal{N}\left(\Lambda_{r}^{i}\left(t_{n}\right), \boldsymbol{u}\left(\cdot, t_{n}\right), \ldots, \boldsymbol{u}\left(\cdot, t_{n-m-w}\right), \boldsymbol{Z}\left(t_{n}\right), \ldots, \boldsymbol{Z}\left(t_{n-m-w}\right), t_{n}\right) \\
\mathcal{L}_{0, \boldsymbol{\Lambda}}\left(t_{n}\right) & =\left(\left\{\mathcal{L}_{0, \Lambda_{r}^{1}}\left(t_{n}\right)\right\}_{r},\left\{\mathcal{L}_{0, \Lambda_{r}^{2}}\left(t_{n}\right)\right\}_{r}\right), \\
\mathcal{L}_{0, \boldsymbol{Z}}\left(t_{n}\right) & =\mathbb{S}\left(t_{n+1}\right) \mathcal{L}_{0, \boldsymbol{\Lambda}}\left(t_{n}\right)
\end{aligned}\right.
$$

These errors are analyzed at the irregular points in the general case of a ADER- $K$ scheme. These points are chosen as they necessitate a particular treatment which requires to change the local error analysis that could be done for a regular point. For a regular point, the analysis is simply the same as in a homogeneous medium. The example of the left irregular point $x_{I_{L}}$ and the right one $x_{I_{R}}$ is chosen because they are irregular points regardless of the choice of the even integer $K$.

Property 1. Let us assume sufficiently smooth initial data, so that the local truncation error for the ADER-K scheme in a homogeneous domain is $\mathcal{O}\left(\Delta x^{K}\right)$. We consider the orders $q=$ $\{3,5\}$ and we set $q_{T}=q_{U} / 4$ so that $\mathbb{M}^{q}$ in $(25)$ is a square matrix. Then, considering the approximations 1, 2 and 3, the local errors (53) satisfy

$$
\left\{\begin{array}{l}
\mathcal{L}_{0, \boldsymbol{u}}\left(x_{I_{L}}, t_{n}\right)=\mathcal{O}\left(\Delta x^{\min (K+1, q)}\right), \\
\mathcal{L}_{0, \boldsymbol{u}}\left(x_{I_{R}}, t_{n}\right)=\mathcal{O}\left(\Delta x^{\min (K+1, q)}\right), \\
\mathcal{L}_{0, \Lambda_{r}^{i}}\left(t_{n}\right)=\mathcal{O}\left(\Delta x^{\min \left(q_{I}, q_{D}+1\right)}\right)+\mathcal{O}\left(\Delta x^{q-1}\right) \sum_{m=0}^{q_{I}-2}\left(\kappa_{r}^{i}\right)^{n-m} \quad\left(i=1,2, r=1, \ldots, N_{R}\right), \\
\mathcal{L}_{0, \boldsymbol{Z}}\left(t_{n}\right)=\left(\left\{\mathcal{O}\left(\Delta x^{\min \left(q, q_{I}, q_{D}+1\right)}\right)\right\}_{1 \leq j \leq N_{R}},\left\{\mathcal{O}\left(\Delta x^{\min \left(q-1, q_{I}, q_{D}+1\right)}\right)\right\}_{N_{R}+1 \leq j \leq 2 N_{R}}\right)
\end{array}\right.
$$

Proof. See Appendix A.

Even if the one lacks of a theoretical stability property for the proposed scheme, we assume that, if applicable, the global error would be in agreement with the analysis of the local error and corresponds to the accumulation of the latter over iterations. Accordingly, a cumulative error at time $t_{n}$ can be found from Proposition 1 by repeating the scheme $\mathcal{O}\left(\frac{1}{\Delta t}\right)$ times. In numerical experiments, we are interested in measurements of global errors on $\boldsymbol{u}$ only. Consequently, in a second step of the analysis we evaluate the effect of the cumulative error regarding $\boldsymbol{Z}$ on the local error regarding $\boldsymbol{u}$. Therefore, the final local error on $\boldsymbol{u}$ after two time steps is defined by:

$$
\left\{\begin{array}{l}
\mathcal{L}_{\boldsymbol{u}}\left(x_{I_{L}}, t_{n}\right)=\boldsymbol{u}\left(x_{I_{L}}, t_{n+2}\right)-\mathcal{H}_{L}\left(\boldsymbol{u}\left(\cdot, t_{n+1}\right)+\mathcal{L}_{0, \boldsymbol{u}}\left(\cdot, t_{n}\right), \boldsymbol{Z}\left(t_{n+1}\right)+\frac{1}{\Delta t} \mathcal{L}_{0, \boldsymbol{Z}}\left(t_{n}\right)\right), \\
\mathcal{L}_{\boldsymbol{u}}\left(x_{I_{R}}, t_{n}\right)=\boldsymbol{u}\left(x_{I_{R}}, t_{n+2}\right)-\mathcal{H}_{R}\left(\boldsymbol{u}\left(\cdot, t_{n+1}\right)+\mathcal{L}_{0, \boldsymbol{u}}\left(\cdot, t_{n}\right), \boldsymbol{Z}\left(t_{n+1}\right)+\frac{1}{\Delta t} \mathcal{L}_{0, \boldsymbol{Z}}\left(t_{n}\right)\right),
\end{array}\right.
$$

and one arrives at the following result. 
Property 2. Let us assume that the assumptions in Property 1 are satisfied and introduce the cumulative error $\mathcal{E}\left(t_{n}\right)=\frac{1}{\Delta t} \mathcal{L}_{\boldsymbol{u}}\left(x_{i}, t_{n}\right)$. Then $\mathcal{E}\left(t_{n}\right)=\mathcal{O}\left(\Delta x^{\delta}\right)$ is obtained with the parameter $\delta$ being given by

$$
\delta=\min \left(K, q-1, q_{I}-1, q_{D}\right) \quad \text { for } q=\{3,5\} .
$$

Proof. See Appendix B.

If stability holds then we expect the cumulative error $\mathcal{E}\left(t_{n}\right)$ to be consistent with the global error. Considering Property 2 and the above discussion on the numerical stability, then one can conclude that a third-order accuracy can be reached but the fourth-order accuracy of the ADER-4 scheme cannot be recovered. In the numerical experiments of Section 5.1, global errors are measured and they are found to be in agreement with the analysis of the local error provided in Property 2. Note that, even if Property 2 is relative to the $1 \mathrm{D}$ case, the numerical experiments of Section 5.2 suggest that similar results also hold in the 2D case.

Lastly, in the non-resonant case, no auxiliary variables need to be defined and $\boldsymbol{Z}\left(t_{n}\right)=\mathbf{0}$ can be set in (18). In this case, the integration and derivation steps of Section 3.6 are irrelevant so that the associated parameters $q_{I}$ and $q_{D}$ can be removed from the estimates of Property 2. As a consequence, the error $\mathcal{E}\left(t_{n}\right)=\mathcal{O}\left(\Delta x^{\min (K, q-1)}\right)$ for all $q$ is recovered, which meets the result proven in [14] for $K=2$.

\section{Numerical modeling in 2D: an overview}

\subsection{Setting and implementation}

In this section, we formally extend the $2 \mathrm{D}$ model (7) to a configuration with a curved enlarged interface defined by two parallel curves $\Gamma_{j}=\Gamma_{j}\left(x_{1}(s), x_{2}(s)\right)$, with $j=0,1$ and $s$ being the associated curvilinear abscissa, see Figure 3. The solution is defined in the domains on each side, which are denoted as $\Omega_{0}$ and $\Omega_{1}$, while as previously no physical field is defined in the interspace between $\Gamma_{0}$ and $\Gamma_{1}$ of width $a$. The jump conditions in (7), which are expressed in Cartesian coordinates are directly transposed in the local frame defined by the normal and tangent vectors $\boldsymbol{\nu}$ and $\boldsymbol{\tau}$ at the interfaces, see Remark 1:

$$
\begin{cases}\partial_{t} \boldsymbol{v}(\boldsymbol{x}, t)=-\frac{1}{\rho_{m}} \boldsymbol{\nabla} p(\boldsymbol{x}, t) & \left(\boldsymbol{x} \in \Omega_{0} \cup \Omega_{1}\right) \\ \partial_{t} p(\boldsymbol{x}, t)=-\frac{1}{\chi_{m}} \operatorname{div} \boldsymbol{v}(\boldsymbol{x}, t) & \left(\boldsymbol{x} \in \Omega_{0} \cup \Omega_{1}\right) \\ \partial_{t} G_{r}(s, t)=-\omega_{r}^{2} J_{r}(s, t)+\left(c_{m} \alpha_{r}\right)^{2}\langle\Delta \operatorname{div} \boldsymbol{v}(\boldsymbol{x}, t)\rangle_{a} & \left(s \in \mathbb{R}, r=1, \ldots, N_{R}\right) \\ \partial_{t} J_{r}(s, t)=G_{r}(s, t) & \left(s \in \mathbb{R}, r=1, \ldots, N_{R}\right) \\ \llbracket p \rrbracket_{a}=B_{1}\left\langle\partial_{\nu} p\right\rangle_{a}+B_{2}\left\langle\partial_{\tau} p\right\rangle_{a} & (s \in \mathbb{R}) \\ \llbracket v_{1} \rrbracket_{a}=C_{11}\left\langle\partial_{\nu} v_{\nu}\right\rangle_{a}+C_{12}\left\langle\partial_{\tau} v_{\nu}\right\rangle_{a}+C_{22}\left\langle\partial_{\tau} v_{\tau}\right\rangle_{a}+h \alpha_{0}\langle\operatorname{div} \boldsymbol{v}\rangle_{a}-h \sum_{r=1}^{N_{R}} J_{r} & (s \in \mathbb{R})\end{cases}
$$


where $v_{\nu}=\boldsymbol{v} \cdot \boldsymbol{\nu} /\|\boldsymbol{\nu}\|, v_{\tau}=\boldsymbol{v} \cdot \boldsymbol{\tau} /\|\boldsymbol{\tau}\|$ and $\partial_{\nu} f=\boldsymbol{\nabla} f \cdot \boldsymbol{\nu} /\|\boldsymbol{\nu}\|, \partial_{\tau} f=\boldsymbol{\nabla} f \cdot \boldsymbol{\tau} /\|\boldsymbol{\tau}\|$. Moreover, $\llbracket f \rrbracket_{a}$ and $\langle f\rangle_{a}$ stand for the $2 \mathrm{D}$ version of the jump and the mean value of $f$ at the enlarged interface respectively.

Remark 6. In the proposed extension (57) of the model (7) to a configuration involving a curved enlarged interface, we have chosen to keep the term $\langle\operatorname{div} \boldsymbol{v}\rangle_{a}$ as such as it has a clear interpretation relatively to a homogenization process, see (3) and [22]. However, we do not claim that (57) is a homogenized model and our objective is rather to show that such a model can be efficiently handled using the proposed approach.

As previously, we use the ADER-4 scheme with a uniform Cartesian grid with $\boldsymbol{u}_{i, j}^{n}$ denoting the numerical value of the solution $\boldsymbol{u}=\left(p, v_{1}, v_{2}\right)^{\top}$ at the point $\left(x_{i}=i \Delta x, x_{j}=j \Delta x\right)$ and time $t_{n}$. In this framework, the approach adopted is as in 1D: the irregular points are detected and phantom values are computed at the grid points that are located in between $\Gamma_{1}$ and $\Gamma_{0}$ and used by the stencil. For $Q$ being such a point, let $\Omega^{+}$and $\Omega^{-}$denote the closest domain and the farthest one respectively (there is no ambiguity in these definitions as the width of the stencil is systematically chosen to be smaller than the width $a$ of the enlarged interface). Then $Q^{ \pm}$denotes the orthogonal projection of $Q$ on $\Gamma^{ \pm}=\partial \Omega^{ \pm}$(see Figure 3).

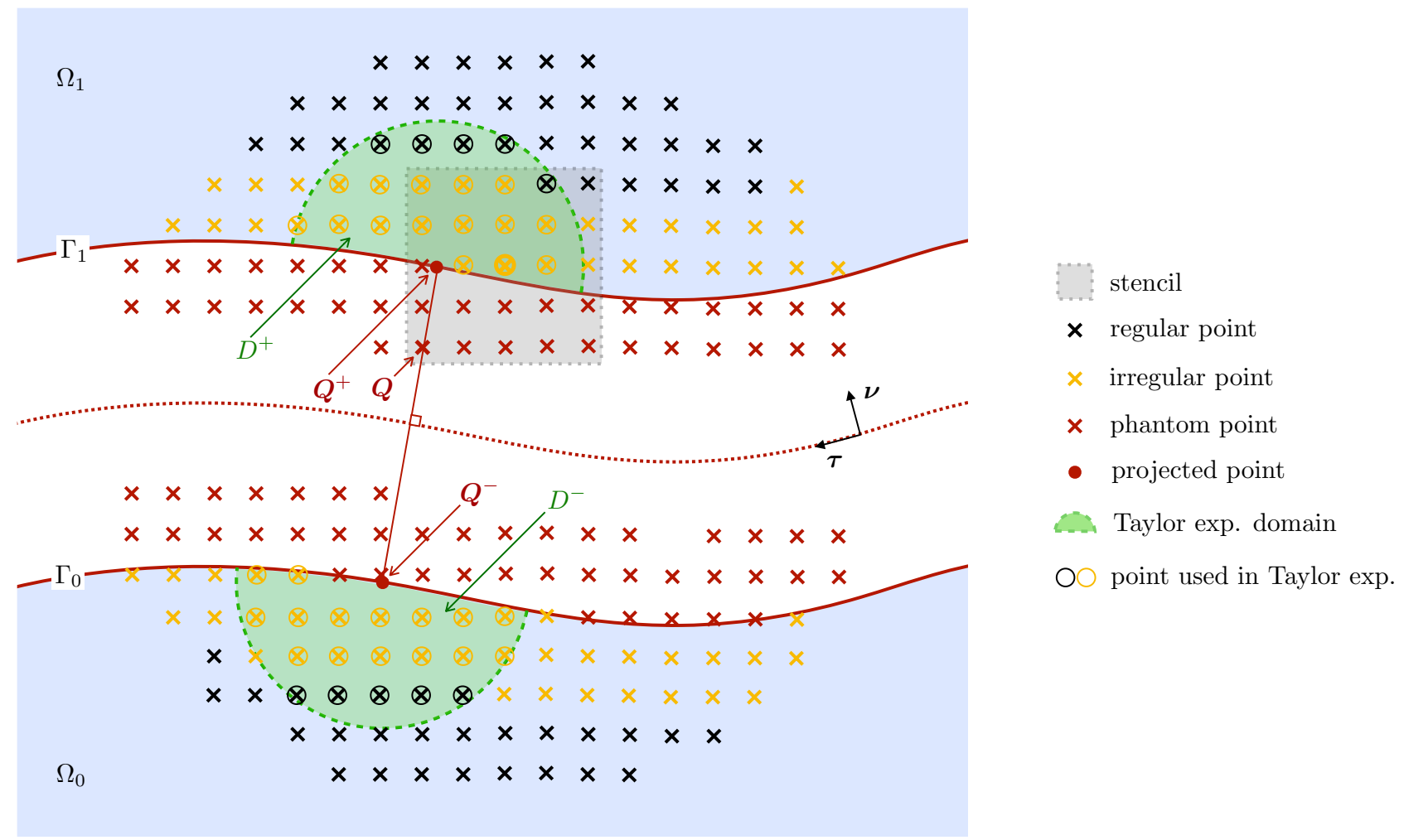

Figure 3: Smooth enlarged interface of width $a$ that separates the domains $\Omega_{0}$ and $\Omega_{1}$ in 2D. A phantom value is sought at the point $Q$ whose orthogonal projections onto the interfaces $\Gamma_{0}$ and $\Gamma_{1}$ are denoted as $Q^{-}, Q^{+}$ respectively.

Extending to the 2D case the definition (15) and (22), a phantom value $\boldsymbol{u}^{*}$ at the point $Q$ is defined as a smooth expansion of the value $\boldsymbol{U}_{+}^{q}\left(t_{n}\right)$ of the solution on the $\Gamma^{+}$interface at the time step $t_{n}$. As in (22), this computation relies on a matrix $\mathbb{T}^{q}\left(Q, Q^{+}\right)$of the polynomial 
forms of the 2D Taylor expansion between the points $Q$ and $Q^{+}$. Moreover, the vectors $\boldsymbol{U}_{ \pm}^{q}\left(t_{n}\right)$ of size $3(q+1)(q+2) / 2$ contain the $2 \mathrm{D}$ derivatives of $\boldsymbol{u}\left(Q^{ \pm}, t_{n}\right)$ up to the chosen order $q$.

\subsection{Principal features of the implementation}

To compute the phantom values, high-order jump conditions relating $\boldsymbol{U}_{+}^{q}\left(t_{n}\right)$ and $\boldsymbol{U}_{-}^{q}\left(t_{n}\right)$ have to be used and these are obtained by differentiating in time the original jump conditions, as it was done in Section 3.4. However, in 2D, high-order derivatives with respect to the curvilinear abscissa $s$ has to be considered as well. This leads to $(q+1)(q+2)$ equations overall. From there, the number of terms in the vectors $\boldsymbol{U}_{ \pm}^{q}\left(t_{n}\right)$ can be reduced based on $q(q+1) / 2$ compatibility conditions that can be deduced from the condition $\boldsymbol{\nabla} \wedge \boldsymbol{v}=\mathbf{0}$, which holds outside the support of the external sources considered. The under-determined system of high-order jump conditions so obtained is then solved in the least-squares sense using Singular Value Decomposition.

Next, 2D Taylor expansions are written out at the grid points that are contained in the domain $D^{+}$(resp. $D^{-}$) defined as the intersection of the disk centered at $Q^{+}$(resp. $Q^{-}$) with the domain $\Omega^{+}$(resp. $\Omega^{-}$), see Figure 3 . This allows to compute $\boldsymbol{U}_{+}^{q}\left(t_{n}\right)$ and finally to derive a formula for the numerical approximation $\left(\boldsymbol{u}^{*}\right)_{i, j}^{n}$ of the phantom values, as in Approximation 1 , by neglecting the Taylor remainders and the derivatives of order $q+1$. This procedure constitutes an extension of the approach developed in [15] for non-resonant interface problems.

Unlike the non-resonant case, it remains however to compute the numerical approximations $J_{r}^{n}, G_{r}^{n}$ of the auxiliary variables $J_{r}(s, t)$ and $G_{r}(s, t)$ along the enlarged interface. This is achieved by performing numerical integration and numerical differentiation, which extends Approximation 3 in 2D. Note that we limit ourself to $q=3$ because when $q>3$ the featured derivatives with respect to the curvilinear abscissa $s$ involve spatial derivatives of the auxiliary variables. Each spatial derivative would be a new auxiliary variable satisfying a new ordinary equation in time. This can be done but we prefer to avoid such technicalities given the satisfying numerical results already obtained for $q=3$. In terms of memory requirements, the computation of the auxiliary variables requires the storage of the vector $\boldsymbol{\Psi}$ defined in (52) for the $1 \mathrm{D}$ case at each orthogonal projection point $Q^{ \pm}$.

\section{Numerical experiments}

5.1. $1 D$ case

\begin{tabular}{|c|c|c|}
\hline$B_{1}(\mathrm{~m})$ & $C_{11}(\mathrm{~m})$ & $h(\mathrm{~m})$ \\
\hline \hline 3.2058 & 0.7558 & 2 \\
\hline
\end{tabular}

\begin{tabular}{|c|c|c|c|c|c|c|}
\hline$r$ & 0 & 1 & 2 & 3 & 4 & 5 \\
\hline \hline$\omega_{r}\left(\mathrm{rad} \cdot \mathrm{s}^{-1}\right)$ & & 300 & 450 & 600 & 750 & 900 \\
\hline$\alpha_{r}$ & 0.314 & 0.462 & 0.4 & 0.2 & 0.1 & 0.1 \\
\hline
\end{tabular}

Table 4: Interface parameters for the 1D model (9).

\begin{tabular}{|c|c|c|c|c|c|c|}
\hline$\rho_{m}\left(\mathrm{~g} \cdot \mathrm{m}^{-3}\right)$ & $c_{m}\left(\mathrm{~m} \cdot \mathrm{s}^{-1}\right)$ & $f_{s}(\mathrm{~Hz})$ & $x_{s}(\mathrm{~m})$ & $a(\mathrm{~m})$ & $\Delta x(\mathrm{~m})$ & $\zeta$ \\
\hline 1000 & 1500 & 30 & -9 & 6 & 1.5 & 0.95 \\
\hline
\end{tabular}

Table 5: Matrix properties and numerical parameters.

This section aims at validating the numerical method described in Section 3 for the $1 \mathrm{D}$ case and assessing the effects of the resonances for the model considered. The chosen constitutive 
parameters and numerical ones are provided in the Tables 4 and 5 considering a number $N_{R}=5$ of resonant frequencies. The time step follows from the CFL condition $\Delta t=\zeta \Delta x / c_{m}$ taken for $\zeta=0.95$. The initial conditions are:

$$
\boldsymbol{u}(x, 0)=\left(-\rho_{m},-1 / c_{m}\right)^{\top} g\left(x-x_{s}\right),
$$

where

$$
g(x)= \begin{cases}\sum_{k=0}^{3} A_{k} \sin \left(2^{k} x\right) & \text { if }-\frac{c_{m}}{f_{s}} \leq x \leq 0 \\ 0 & \text { otherwise, }\end{cases}
$$

with $A_{0}=1, A_{1}=-21 / 32, A_{2}=63 / 768$, and $A_{3}=-1 / 512$, which entails that $g$ is of class $C^{6}$. For the chosen values of $c_{m}$ and $\Delta x$ in Table 5 then the number of grid nodes is approximately 33 per wavelength at the central frequency $f_{s}$. Moreover, the initialization point $x_{s}$ is chosen so that the support of the initial time conditions (58) does not intersect the enlarged interface. The corresponding initial pressure and its Fourier spectrum are shown in Figure 4. The final simulation time is chosen so that the wave has not hit yet the boundaries of the computational domain.

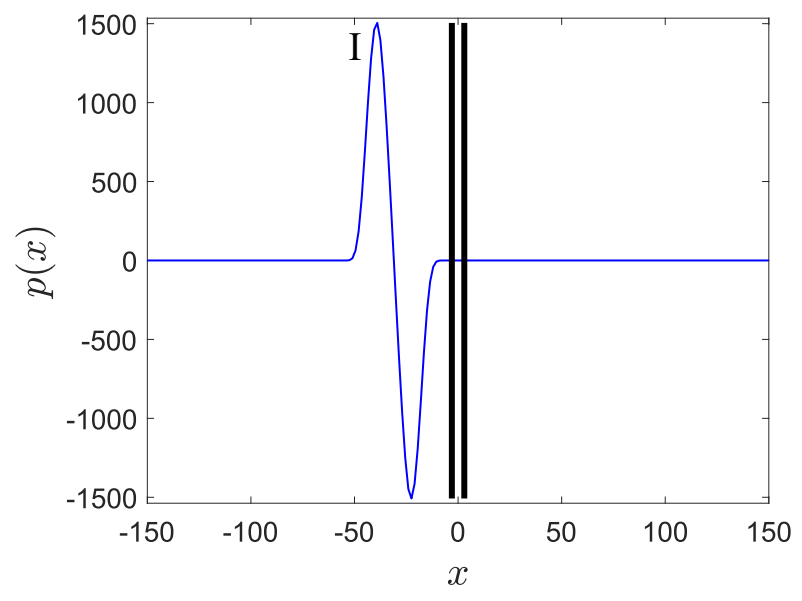

(a) Waveform

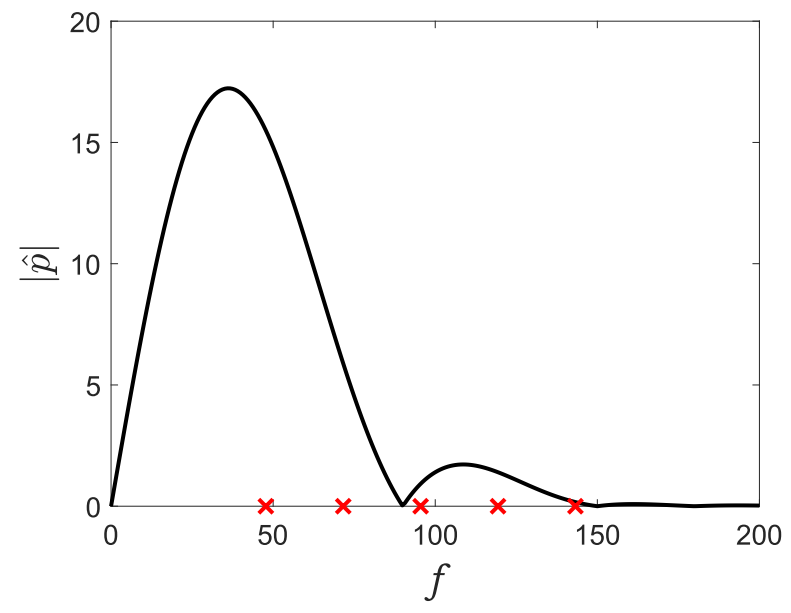

(b) Fourier spectrum

Figure 4: Initial condition (58): (a) pressure field $p$ and (b) associated spectrum with the red crosses indicating the five resonant frequencies $f_{r}=\omega_{r} / 2 \pi$ considered.

Figure 5(a) displays the associated acoustic pressure $p$ computed at the final simulation time $t_{\mathrm{f}}=94.05 \mathrm{~ms}$, together with a semi-analytical solution $p_{\text {ref }}$, which is derived in Appendix C. The discrepancy between the two solutions is quantified by defining a global relative error at the final simulation time $t_{\mathrm{f}}$ as follows:

$$
\epsilon_{p}\left(t_{\mathrm{f}}\right)=\frac{\left\|p_{\mathrm{ref}}\left(\cdot, t_{\mathrm{f}}\right)-p\left(\cdot, t_{\mathrm{f}}\right)\right\|_{L^{2}\left(\Omega_{\mathrm{obs}}\right)}}{\left\|p_{\mathrm{ref}}\left(\cdot, t_{\mathrm{f}}\right)\right\|_{L^{2}\left(\Omega_{\mathrm{obs}}\right)}}
$$

where $\Omega_{\mathrm{obs}}=\left[x_{\mathrm{ini}} ; x_{\mathrm{end}}\right] \backslash[-a / 2 ; a / 2]$, with $x_{\text {ini }}$ and $x_{\text {end }}$ the left and right boundaries of the computational domain. One measures $\epsilon_{p}\left(t_{\mathrm{f}}\right)=1.5 \cdot 10^{-2}$ when $q=3,\left(q_{D}, q_{I}\right)=(2,3)$ and $\lambda / \Delta x=33$ for the characteristic wavelength $\lambda=c_{m} / f_{s}$. The relative error $\epsilon_{p}\left(t_{\mathrm{f}}\right)$ is represented 


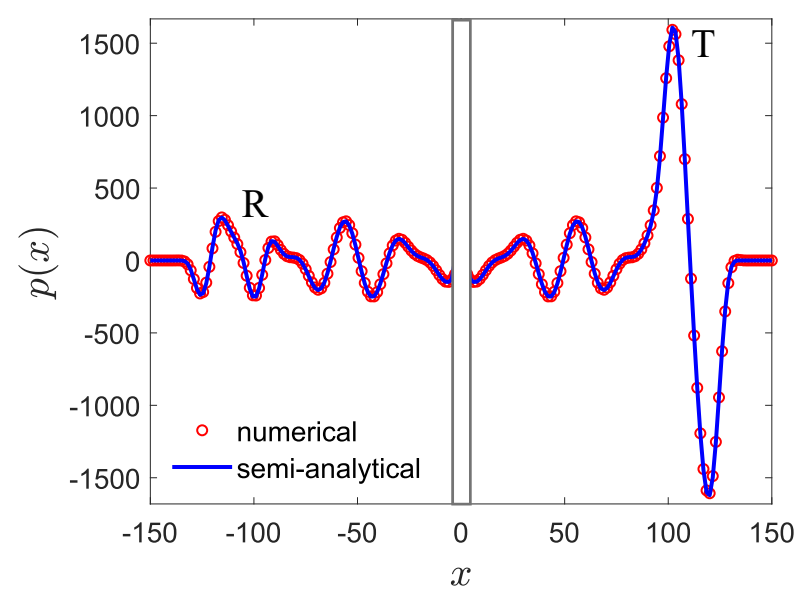

(a) $p_{\text {ref }}$ (blue) and $p$ (red) at the final simulation time $t_{\mathrm{f}}=94.05 \mathrm{~ms}$. R: reflected wave, $\mathrm{T}$ : transmitted wave.

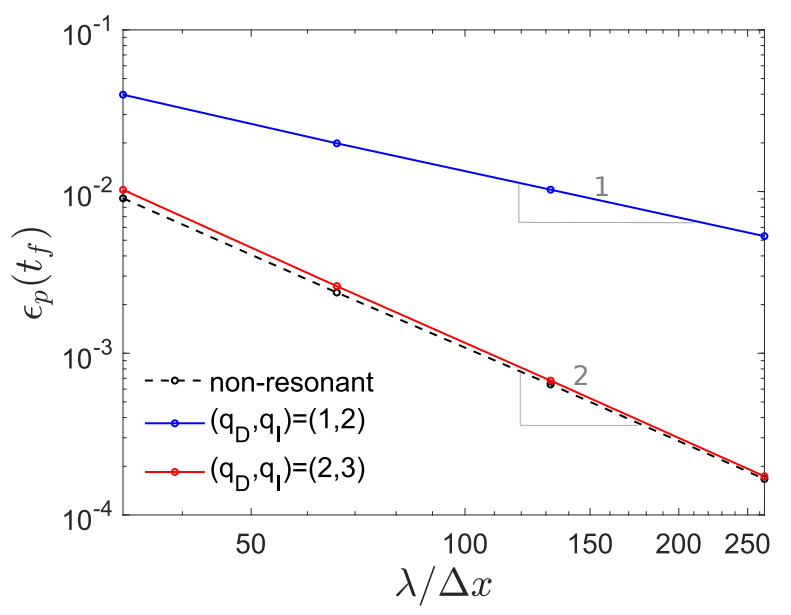

(b) Relative error $\epsilon_{p}\left(t_{\mathrm{f}}\right)$ in a log-log scale.

Figure 5: Numerical results for pressure field $p$ in the 1D case: comparison with a semi-analytical solution $p_{\text {ref }}$.

as a function of $\lambda / \Delta x$ whose slope in a log-log scale graph characterizes the global order of the scheme, see Figure 5(b) for $q=3$ and two values of $\left(q_{D}, q_{I}\right)$. The errors obtained in the non-resonant case, with $\alpha_{r}=0$ for $r=0, \ldots, 5$, are included in Fig. 5(b) for comparison. It is seen that setting $\left(q_{D}, q_{I}\right)=(1,2)$ yields order 1 , which is a drop in accuracy compared to the non-resonant case for which the global error considered is of order 2 . The choice $\left(q_{D}, q_{I}\right)=(2,3)$ allows to recover this order with comparable accuracy.

\begin{tabular}{|l||l|l|l|l|l|}
\hline & $q_{I}$ & 2 & 3 & 4 & 5 \\
$q_{D}$ & 2 & & & \\
\hline \hline 1 & 1 & 1 & 1 & 1 \\
\hline 2 & 1 & 2 & 2 & 2 \\
\hline 3 & 1 & 2 & 2 & 2 \\
\hline 4 & 1 & 2 & 2 & \\
\hline
\end{tabular}

\begin{tabular}{|l||c|c|c|c|}
\hline \multicolumn{1}{r||}{$q_{I}$} & 2 & 3 & 4 & 5 \\
$q_{D}$ & & & & \\
\hline \hline 1 & 1 & 1 & 1 & 1 \\
\hline 2 & 1 & 2 & 2 & 2 \\
\hline 3 & 1 & 2 & 3 & 3 \\
\hline 4 & 1 & 2 & 3 & \\
\hline
\end{tabular}

Table 6: Convergence measurements in the 1D case: accuracy orders for $q=3$ (left) and $q=5$ (right).

Table 6 reports the orders of accuracy measured using the global error metric $\epsilon_{p}\left(t_{\mathrm{f}}\right)$ for $q=3$ and $q=5$ depending on the chosen values $\left(q_{D}, q_{I}\right)$. The parameter $q_{T}$ is chosen to be the minimal value given by Table 3 for each value of $\left(q_{D}, q_{I}\right)$. These orders, as well as Figure 5(b), are obtained with the relative position of the interfaces within the Cartesian grid being kept while the ratio $\lambda / \Delta x$ is increased. Even if the one lacks of a theoretical stability property for the proposed scheme, these numerical results are in agreement with the analysis of the local truncation error in Property 2. Note that, in practice, for the largest values of $\left(q_{D}, q_{I}\right)$ considered then the Taylor expansion parameter $q_{T}$ must be increased to maintain the numerical stability. Doing so, the matrix $\mathbb{M}^{q}$ is no longer a square matrix so that Property 2 does not apply anymore. However the corresponding orders measured remain compatible with the estimates of Property 2. The right bottom boxes are not filled in Table 6 because, the associated values $q_{T}$ being too large, the use of the pseudo-inverse of the matrix $\mathbb{M}^{q}$ yields unacceptable numerical errors. 


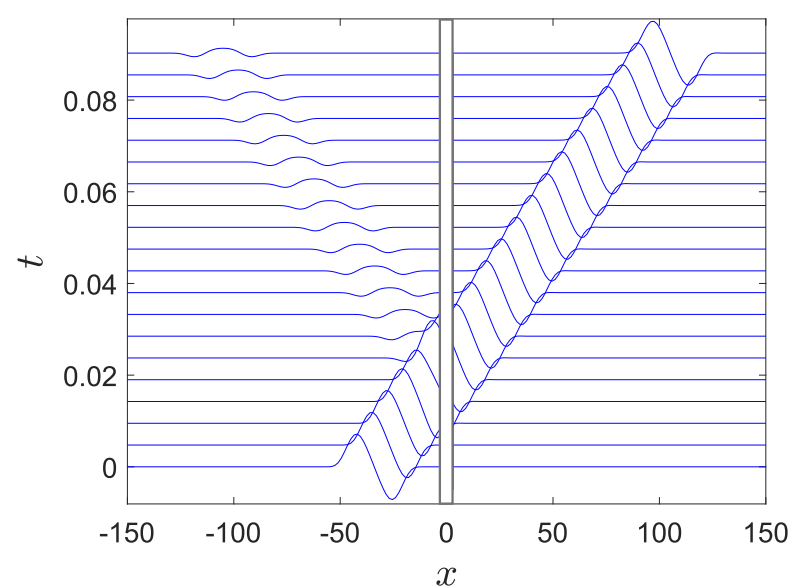

(a) Without resonances $\left(\alpha_{r}=0\right)$

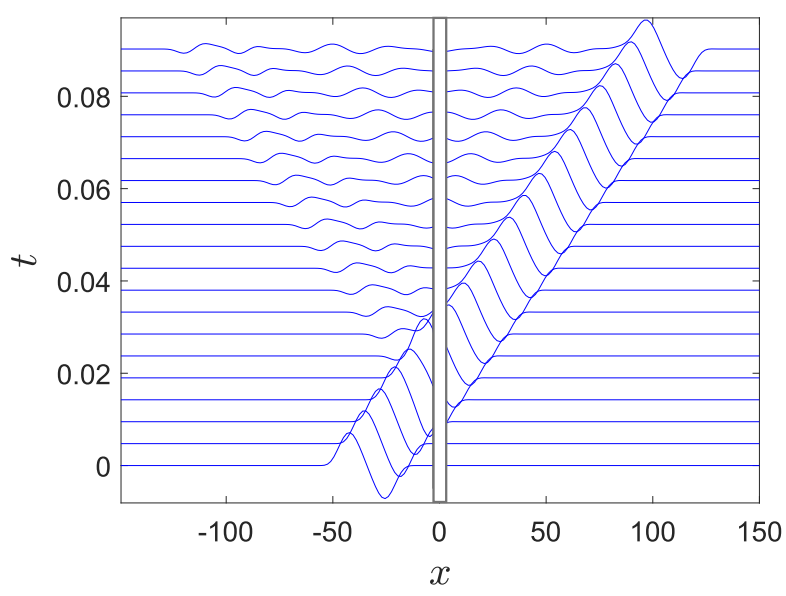

(b) With resonances $\left(\alpha_{r}\right.$ from Table 4$)$

Figure 6: Seismogram of the acoustic pressure p field.

After this validation of the one-dimensional method, we can investigate the effects of the resonances. Figure 6 displays the seismograms of the pressure field with resonances in the jump conditions and without (i.e. when $\alpha_{r}=0$ for $r=0, \ldots, 5$ ). This figure highlights the resonant behavior of the wavefield, with energy being radiated away from the enlarged interface long after the passing of the incident wave. This is a consequence of the frequency dependence of the parameter $\mathcal{D}(\omega)$ in (5) that intervenes in the definition of the jump conditions of the model (3) considered.

\section{2. $2 D$ case}

In this section, we validate the method dicussed in Section 4 on three test cases: i) incident plane wave at normal incidence on a plane enlarged interface, ii) slanted incident plane wave on a tilted enlarged interface, and iii) incident plane wave on a circular enlarged interface. Semi-analytical solutions are computed in these three cases, see Appendix C and Appendix D. The initial conditions are

$\boldsymbol{u}(\boldsymbol{x}, 0)=\left(p, v_{1}, v_{2}\right)^{\top}(\boldsymbol{x}, 0)=-\left(\rho_{m}, \cos \theta_{I} / c_{m}, \sin \theta_{I} / c_{m}\right)^{\top} g\left(\left(x_{1}-x_{s 1}\right) \cos \theta_{I}+\left(x_{2}-x_{s 2}\right) \sin \theta_{I}\right)$,

where $g$ is defined in (59), $\theta_{I}$ is the angle between the direction of propagation of the plane wave and the horizontal axis and the initialization point $\boldsymbol{x}_{s}=\left(x_{s 1}, x_{s 2}\right)$ is chosen so that the support of the initial time conditions does not intersect the enlarged interface. The constitutive and numerical parameters are those of the tables 4 and 5 while the chosen additional interface parameters that are requested in $2 \mathrm{D}$ are reported in Table 7 . The proposed numerical method is implemented taking $q=3$ since, as previously discussed, the cases $q \geq 4$ are very demanding in $2 \mathrm{D}$ and require to handle additional auxiliary variables.

\begin{tabular}{|c|c|c|}
\hline$B_{2}(\mathrm{~m})$ & $C_{12}(\mathrm{~m})$ & $C_{22}(\mathrm{~m})$ \\
\hline \hline 0.284 & 0.284 & 0.4 \\
\hline
\end{tabular}

Table 7: Additional interface parameters for the 2D model (57). 


\subsubsection{Incident plane wave at normal incidence}

First, we consider the case of normal incidence, i.e. $\theta_{I}=0$, with a wave impacting normally a plane enlarged interface, in which case the fields are independent of $x_{2}$. Periodicity conditions are imposed at the bottom and top boundaries of the computational domain. Physically, the problem is $1 \mathrm{D}$, but the full $2 \mathrm{D}$ algorithm is employed and one sets $\left(q_{D}, q_{I}\right)=(2,3)$.

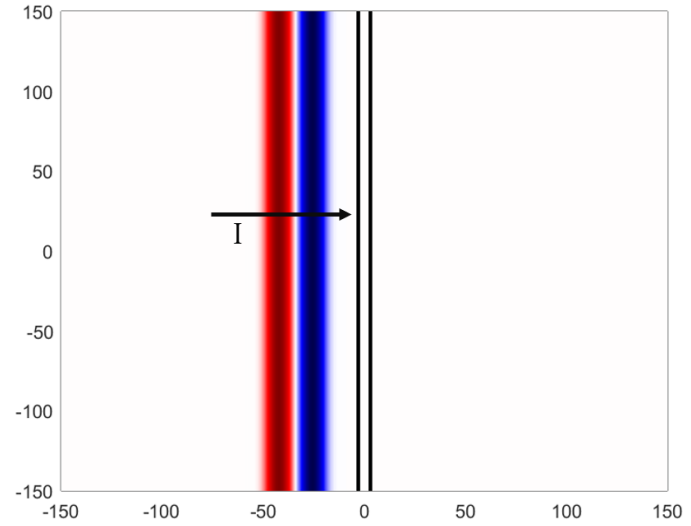

(a) $t=0$

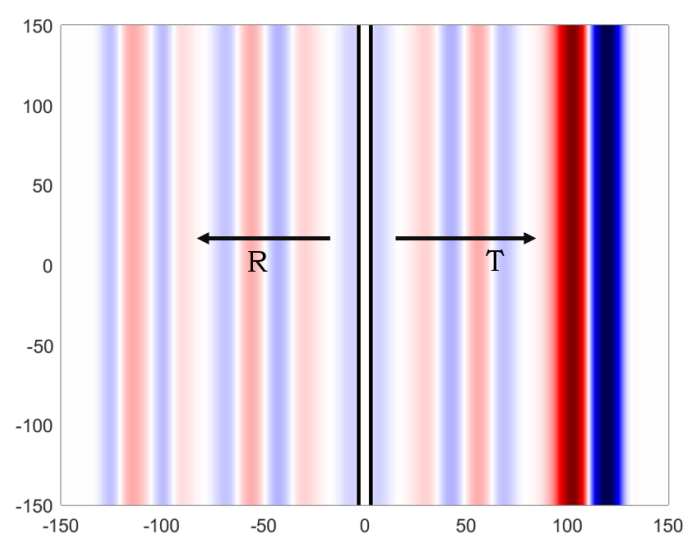

(b) $t_{\mathrm{f}}=94.05 \mathrm{~ms}$

Figure 7: Pressure field $p$ computed for an incident plane wave (I) at normal incidence on the enlarged interface. $\mathrm{R}$ : reflected wave, T: transmitted wave.

Figure 7 displays the pressure field $p$ at the initial time $t=0$, at which the initialization point in $(60)$ is $\boldsymbol{x}_{s}=(-9 \mathrm{~m}, 0 \mathrm{~m})$, and at time $t_{\mathrm{f}}=94.05 \mathrm{~ms}$, while Figure 8 shows the profiles of the solution at $x_{2}=0$. The discrepancy between the numerical and the semi-analytical solutions is comparable with the $1 \mathrm{D}$ results with $\epsilon_{p}\left(t_{\mathrm{f}}\right)=4.5 \cdot 10^{-2}$ for $\lambda / \Delta x=33$. Table 8 reports the measured orders of convergence, which are the same than in 1D.

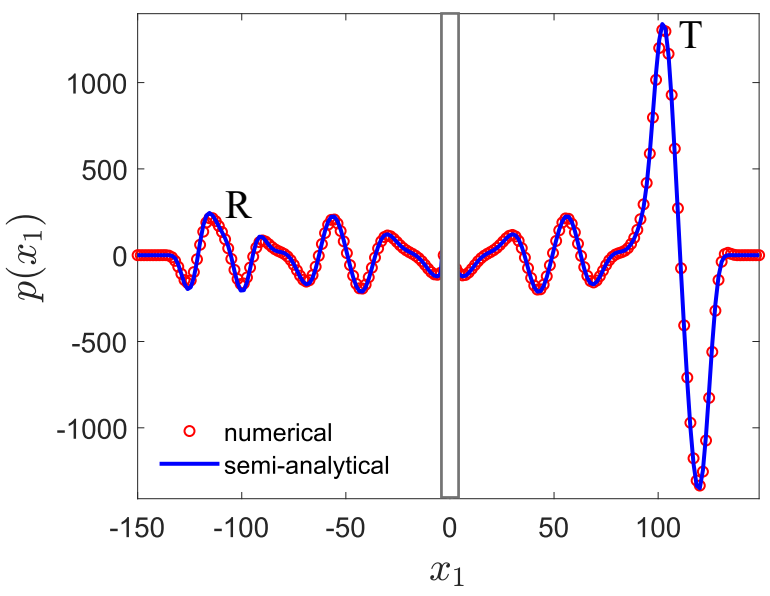

Figure 8: Incident plane wave at normal incidence in 2D: semi-analytical and numerical pressure fields $p_{\text {ref }}$ (blue) and $p$ (red) at $x_{2}=0$ and for the final simulation time $t_{\mathrm{f}}=94.05 \mathrm{~ms}$. 


\begin{tabular}{|l||c|c|c|c|}
\hline \multicolumn{1}{|r||}{$q_{I}$} & 2 & 3 & 4 & 5 \\
\hline$q_{D}$ & & & & \\
\hline \hline 1 & 1 & 1 & 1 & 1 \\
\hline 2 & 1 & 2 & 2 & 2 \\
\hline 3 & 1 & 2 & 2 & 2 \\
\hline 4 & 1 & 2 & 2 & \\
\hline
\end{tabular}

Table 8: Convergence measurements in the 2D case: accuracy orders for $q=3$.

\subsubsection{Slanted incident plane wave on a tilted enlarged interface}

The case of an incident wave at oblique incidence, with $\theta_{I}=10^{\circ}$ in (60), on an enlarged interface tilted from the vertical axis at $-7.2^{\circ}$ is now considered. It allows us to inspect both the dependencies of the jump conditions on $x_{2}$ and the capability of the numerical method to account for the slope of the interfaces on a Cartesian grid. To perform the simulations, one imposes the semi-analytical solution of the problem on the domain boundary.

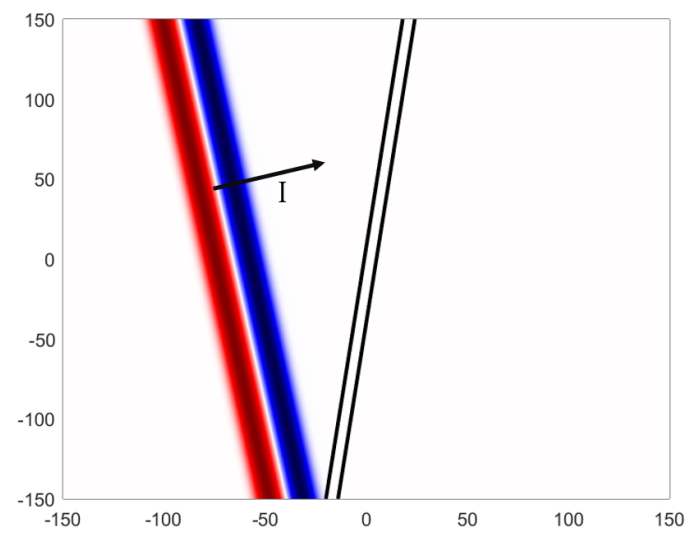

(a) $t=0$

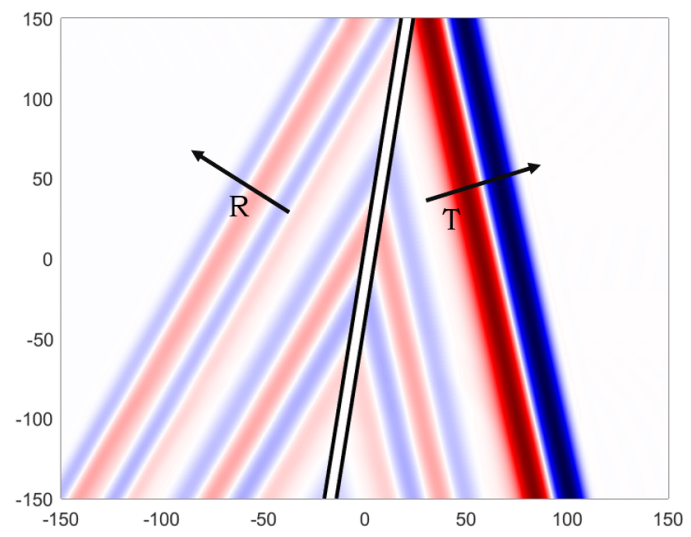

(b) $t_{\mathrm{f}}=84.55 \mathrm{~ms}$

Figure 9: Pressure field $p$ computed for an incident plane wave (I) at oblique incidence on a tilted enlarged interface. $\mathrm{R}$ : reflected wave, $\mathrm{T}$ : transmitted wave.

Figure 9 displays the pressure field computed at the initial time, at which the initialization point in $(60)$ is $\boldsymbol{x}_{s}=(-21 \mathrm{~m},-150 \mathrm{~m})$, and at time $t_{\mathrm{f}}=84.55 \mathrm{~ms}$ when $\left(q_{D}, q_{I}\right)=(2,3)$. Figure 10 compares the reference semi-analytical solution and the numerical one, with $\epsilon_{p}\left(t_{\mathrm{f}}\right)=4.1 \cdot 10^{-2}$ when $\lambda / \Delta x=33$. This result is comparable to that given in Section 5.2.1, which illustrates that the dependency of the jump conditions on $x_{2}$ and the slope of the interfaces are both accurately accounted for by the proposed numerical method.

\subsubsection{Incident plane wave on a circular enlarged interface}

The case of an incident plane wave on a circular enlarged interface is now considered. This example allows to inspect the capability of the method to take into account a curved enlarged interface. A semi-analytical solution is derived in Appendix D in the case $B_{2}=C_{12}=\alpha_{0}=0$. Consequently, in this subsection, these interface parameters are set to zero to allow the comparison with the semi-analytical solution. The numerical parameters are $\left(q_{D}, q_{I}\right)=(2,3)$. 


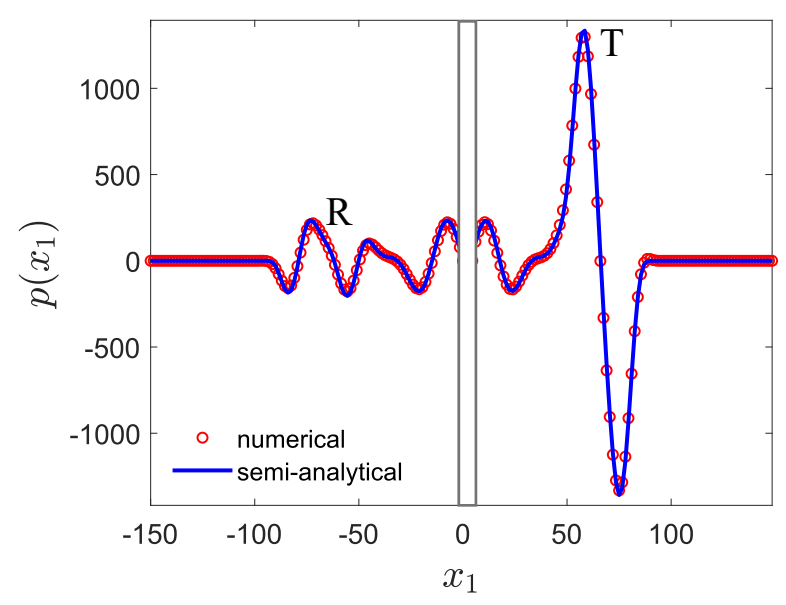

Figure 10: Slanted incident plane wave on a tilted enlarged interface: semi-analytical and numerical pressure fields $p_{\text {ref }}$ (blue) and $p$ (red) at $x_{2}=0$ and for the final simulation time $t_{\mathrm{f}}=84.55 \mathrm{~ms}$.

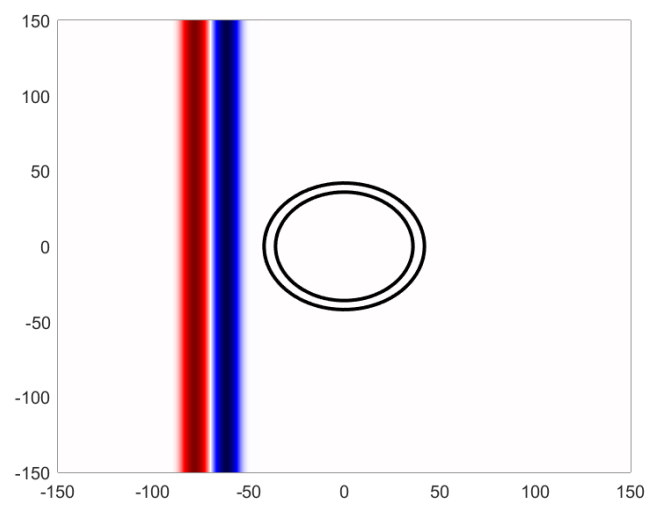

(a) $t=0$

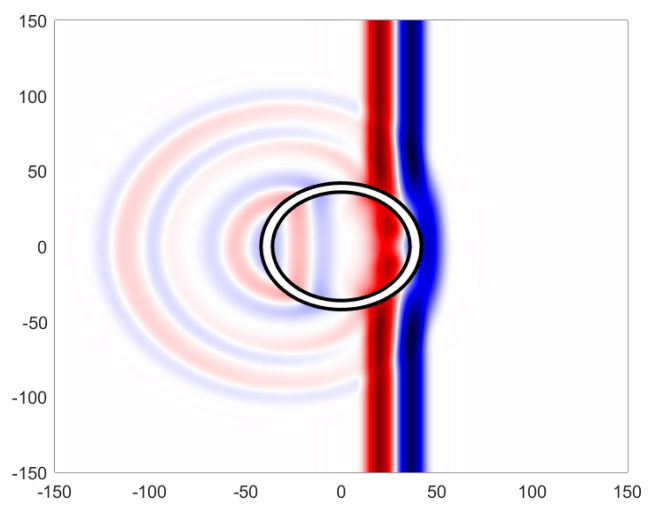

(b) $t_{\mathrm{f}}=63.3 \mathrm{~ms}$

Figure 11: Pressure field $p$ computed for an incident plane wave on a circular enlarged interface.

We consider an incident plane wave with propagation direction along $x_{1}$ and periodicity conditions imposed on the top and bottom boundaries of the computational domain. The pressure field at the initial time, at which the initialization point in (60) is $\boldsymbol{x}_{s}=(-45 \mathrm{~m}, 0 \mathrm{~m})$, and at final simulation time $t_{\mathrm{f}}=63.3 \mathrm{~ms}$ are displayed in Figure 11. The comparison with the semi-analytical solution is reported in Figure 12 at $x_{2}=0$. The measured error is $\epsilon_{p}\left(t_{\mathrm{f}}\right)=2 \cdot 10^{-2}$ when $\lambda / \Delta x=33$, which highlights the satisfying performances of the proposed approach with the circular enlarged interface being accurately handled numerically.

\section{Conclusion}

In this study, our objective was to handle enlarged interfaces characterized by frequencydependent jump conditions and consequently jump conditions that are non-local in time when formulated in the time domain. A time-domain numerical method was proposed and implemented for this type of model. To the best of our knowledge, this constitutes the first effort to simulate transient wave propagation across such resonant meta-interfaces. The proposed 


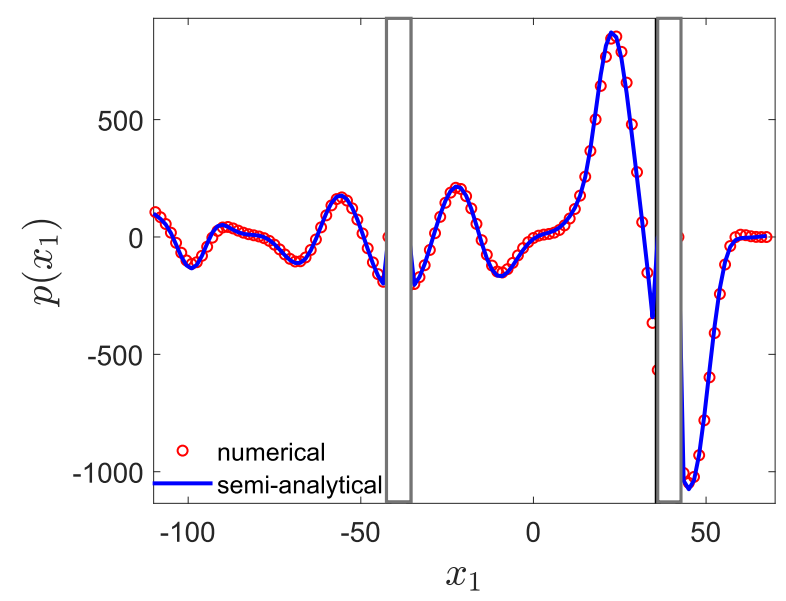

Figure 12: Incident plane wave on a circular enlarged interface in 2D: semi-analytical and numerical pressure fields $p_{\text {ref }}$ (blue) and $p$ (red) at $x_{2}=0$ and for the final simulation time $t_{\mathrm{f}}=63.3$ ms. The vertical black lines denote the position of the enlarged circular interface.

approach relies on the following key points: (i) A set of auxiliary variables is introduced locally along the enlarged interface, which allows to formulate a first-order system in time with jump conditions that are local in time. (ii) An immersed interface method is developed to handle numerically such a system by using a high-order finite differences scheme on a Cartesian grid while offering a subcell resolution through a proper discretization of the enlarged interface. Local error estimates were derived to assess the optimal values of the featured numerical parameters. The proposed numerical method was then illustrated and validated considering 1D and $2 \mathrm{D}$ configurations involving plane waves illuminating straight or curved enlarged interfaces. Moreover, the solutions to these problems were derived and used for quantitative comparisons.

Future directions of work concern the validation of the time-domain interface model considered itself. Indeed, as it has been discussed in the introduction, such resonant meta-interface models can be constructed systematically from the homogenization of a microstructured layer containing inclusions whose constitutive properties have a particular scaling with respect to these of the background medium. With the proposed numerical method at hand then the validity of such effective meta-interface models can be assessed through comparison with full-field simulations. Another perspective concerns the design of meta-interfaces through the optimization of an underlying microstructured medium to reach some objective effective properties. This is of particular interest in view of, e.g., noise reduction by thin resonant metasurfaces $[18,24]$. A promising strategy relies on the topological optimization tools developed in $[9,3]$. 


\section{Appendix A. Proof of Property 1}

Appendix A.1. Estimation of $\mathcal{L}_{0, u}$

$\mathcal{L}_{0, \boldsymbol{u}}$ is defined in (53) for the two irregular points $x_{I_{L}}$ and $x_{I_{R}}$. These local errors can be expressed as:

$$
\begin{aligned}
\mathcal{L}_{0, \boldsymbol{u}}\left(x_{I_{L}}, t_{n}\right)=\boldsymbol{u}\left(x_{I_{L}}, t_{n+1}\right)-\boldsymbol{u}\left(x_{I_{L}}, t_{n}\right) & +\sum_{s=-K / 2}^{0} \sum_{m=1}^{K} \nu_{K, m, s}\left(\mathbb{A} \frac{\Delta t}{\Delta x}\right)^{m} \boldsymbol{u}\left(x_{I_{L}+s}, t_{n}\right) \\
& +\sum_{s=1}^{K / 2} \sum_{m=1}^{K} \nu_{K, m, s}\left(\mathbb{A} \frac{\Delta t}{\Delta x}\right)^{m} \tilde{\boldsymbol{u}}^{*}\left(x_{I_{L}+s}, t_{n}\right),
\end{aligned}
$$

and

$$
\begin{aligned}
\mathcal{L}_{0, \boldsymbol{u}}\left(x_{I_{R}}, t_{n}\right)=\boldsymbol{u}\left(x_{I_{R}}, t_{n+1}\right)-\boldsymbol{u}\left(x_{I_{R}}, t_{n}\right) & +\sum_{s=-K / 2}^{-1} \sum_{m=1}^{K} \nu_{K, m, s}\left(\mathbb{A} \frac{\Delta t}{\Delta x}\right)^{m} \tilde{\boldsymbol{u}}^{*}\left(x_{I_{R}+s}, t_{n}\right) \\
& +\sum_{s=0}^{K / 2} \sum_{m=1}^{K} \nu_{K, m, s}\left(\mathbb{A} \frac{\Delta t}{\Delta x}\right)^{m} \boldsymbol{u}\left(x_{I_{R}+s}, t_{n}\right),
\end{aligned}
$$

where $\tilde{\boldsymbol{u}}^{*}\left(x_{i}, t_{n}\right)$ denotes the phantom value obtained when replacing the numerical values $(\boldsymbol{u}, \boldsymbol{\Lambda}, \boldsymbol{Z})^{n}$ by their exact counterparts in Approximation 1. These fields are assumed to be as smooth as necessary.

To estimate (A.1) and (A.2), one first evaluates the approximation of the phantom values $\tilde{\boldsymbol{u}}^{*}\left(x_{i}, t_{n}\right)$. From Approximation 1, these quantites write:

$$
\begin{cases}\tilde{\boldsymbol{u}}^{*}\left(x_{I_{L}+s}, t_{n}\right)=\mathbb{T}_{i}^{q}\left(-\frac{a}{2}\right)\left(\mathbb{M}^{q}\right)^{-1}\left[\mathcal{U}\left(t_{n}\right)+\mathbb{F}^{q} \mathbb{Q}^{q} \boldsymbol{Z}\left(t_{n}\right)\right] & \text { for } s=1, \ldots, K / 2, \\ \tilde{\boldsymbol{u}}^{*}\left(x_{I_{R}+s}, t_{n}\right)=\mathbb{T}_{i}^{q}\left(\frac{a}{2}\right)\left(\mathbb{C}_{+}^{q}\right)^{-1}\left[\mathbb{C}_{-}^{q}\left(\mathbb{M}^{q}\right)^{-1} \mathcal{U}\left(t_{n}\right)+\mathbb{G}^{q} \mathbb{Q}^{q} \boldsymbol{Z}\left(t_{n}\right)\right] & \text { for } s=-K / 2, \ldots,-1 .\end{cases}
$$

Using (28) and (29), one gets

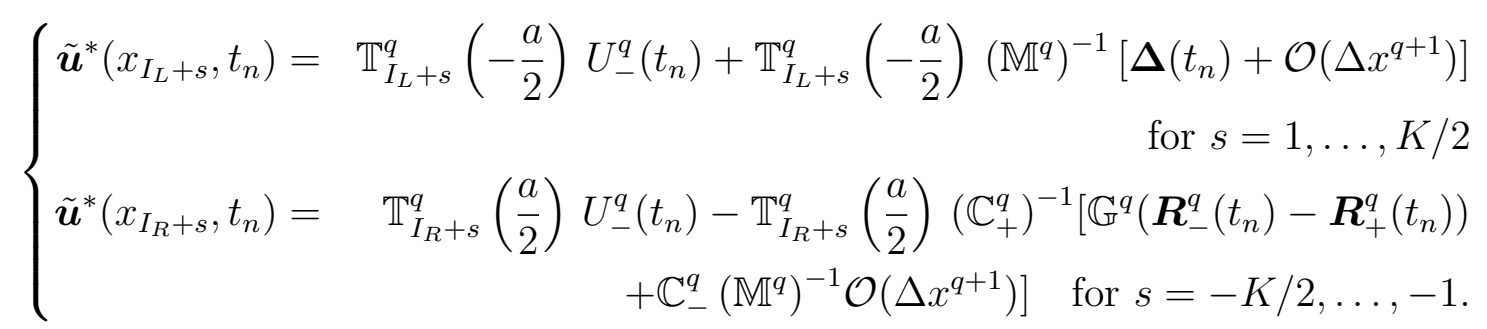

Symbolic computations are then performed using Maple to estimate the leading contributions of the entries of the vectors in (A.4). Doing so provides, for $q=3$ or 5 :

$$
\left\{\begin{array}{l}
\mathbb{T}_{I_{L}+s}^{q}\left(-\frac{a}{2}\right)\left(\mathbb{M}^{q}\right)^{-1} \Delta\left(t_{n}\right)=\mathcal{O}\left(\Delta x^{q}\right), \\
\mathbb{T}_{I_{L}+s}^{q}\left(-\frac{a}{2}\right)\left(\mathbb{M}^{q}\right)^{-1} \mathcal{O}\left(\Delta x^{q+1}\right)=\mathcal{O}\left(\Delta x^{q+1}\right), \\
\mathbb{T}_{I_{R}+s}^{q}\left(\frac{a}{2}\right)\left(\mathbb{C}_{+}^{q}\right)^{-1} \mathbb{G}^{q}\left(\boldsymbol{R}_{-}^{q}\left(t_{n}\right)-\boldsymbol{R}_{+}^{q}\left(t_{n}\right)\right)=\mathcal{O}\left(\Delta x^{q}\right), \\
\mathbb{T}_{I_{R}+s}^{q}\left(\frac{a}{2}\right)\left(\mathbb{C}_{+}^{q}\right)^{-1} \mathbb{C}_{-}^{q}\left(\mathbb{M}^{q}\right)^{-1} \mathcal{O}\left(\Delta x^{q+1}\right)=\mathcal{O}\left(\Delta x^{q+1}\right) .
\end{array}\right.
$$


One notes that the first and third of the above estimates make use of the definition (21) of $\boldsymbol{R}_{ \pm}^{q}\left(t_{n}\right)$, which are expressed in terms of the exact solution, and it is assumed that the latter is sufficiently smooth. Therefore, for $q=3$ or 5 , we finally obtain the following estimate for the approximation of the phantom values:

$$
\tilde{\boldsymbol{u}}^{*}\left(x_{i}, t_{n}\right)=\mathbb{T}_{i}^{q}\left(\mp \frac{a}{2}\right) \boldsymbol{U}_{\mp}^{q}\left(t_{n}\right)+\mathcal{O}\left(\Delta x^{q}\right) .
$$

The top (resp. bottom) sign corresponds to the left (resp. right) case, the order of this approximation being the same for both sides. Consequently, from now on we will only consider $x_{I_{R}}$ to evaluate the order of $\mathcal{L}_{0, \boldsymbol{u}}$ but the final result will apply to any irregular point. We use Taylor expansions and (A.6) for each term of (A.2) to get:

$$
\left\{\begin{array}{l}
\boldsymbol{u}\left(x_{I_{R}+s}, t_{n}\right)=\sum_{\ell=0}^{q} \frac{1}{\ell !}\left(x_{I_{R}+s}-\frac{a}{2}\right)^{\ell} \partial_{x}^{\ell} \boldsymbol{u}\left(\frac{a}{2}, t_{n}\right)+\mathcal{O}\left(\Delta x^{q+1}\right), \\
\tilde{\boldsymbol{u}}^{*}\left(x_{I_{R}+s}, t_{n}\right)=\sum_{\ell=0}^{q} \frac{1}{\ell !}\left(x_{I_{R}+s}-\frac{a}{2}\right)^{\ell} \partial_{x}^{\ell} \boldsymbol{u}\left(\frac{a}{2}, t_{n}\right)+\mathcal{O}\left(\Delta x^{q}\right) .
\end{array}\right.
$$

Since $\Delta t=\mathcal{O}(\Delta x)$ holds from the CFL condition of the ADER-K scheme, then one can write

$$
\mathcal{L}_{0, \boldsymbol{u}}\left(x_{I_{R}}, t_{n}\right)=\mathcal{L}_{0, \boldsymbol{u}}^{0}\left(x_{I_{R}}, t_{n}\right)+\sum_{m=1}^{K} \mathcal{L}_{0, \boldsymbol{u}}^{m}\left(x_{I_{R}}, t_{n}\right)+\mathcal{O}\left(\Delta x^{q}\right),
$$

when defining for $m=1, \ldots, K$ :

$$
\begin{aligned}
& \mathcal{L}_{0, \boldsymbol{u}}^{0}\left(x_{I_{R}}, t_{n}\right)=\boldsymbol{u}\left(x_{I_{R}}, t_{n+1}\right)-\boldsymbol{u}\left(x_{I_{R}}, t_{n}\right) \\
& \mathcal{L}_{0, \boldsymbol{u}}^{m}\left(x_{I_{R}}, t_{n}\right)=\sum_{s=-K / 2}^{+K / 2} \nu_{K, m, s}\left(\mathbb{A} \frac{\Delta t}{\Delta x}\right)^{m} \sum_{\ell=0}^{q} \frac{1}{\ell !}\left(x_{I_{R}+s}-\frac{a}{2}\right)^{\ell} \partial_{x}^{\ell} \boldsymbol{u}\left(\frac{a}{2}, t_{n}\right) .
\end{aligned}
$$

From a Taylor expansion at the order $K$ in time and using (11) in combination with Taylor expansions at the order $(q-m)$ in space, we can write

$$
\begin{aligned}
\boldsymbol{u}\left(x_{I_{R}}, t_{n+1}\right) & =\boldsymbol{u}\left(x_{I_{R}}, t_{n}\right)+\sum_{m=1}^{K} \frac{\Delta t^{m}}{m !}(-1)^{m} \mathbb{A}^{m} \sum_{\ell=0}^{q-m} \frac{1}{\ell !}\left(x_{I_{R}}-\frac{a}{2}\right)^{\ell} \partial_{x}^{m+\ell} \boldsymbol{u}\left(\frac{a}{2}, t_{n}\right) \\
& +\mathcal{O}\left(\Delta x^{q+1}\right)+\mathcal{O}\left(\Delta x^{K+1}\right) .
\end{aligned}
$$

Combining (A.9) and (A.10), we can rewrite $\mathcal{L}_{0}\left(x_{I_{R}}, t_{n}\right)$ as follows:

$$
\begin{aligned}
\mathcal{L}_{0, \boldsymbol{u}}^{0}\left(x_{I_{R}}, t_{n}\right) & =\sum_{m=1}^{K} \frac{\Delta t^{m}}{m !}(-1)^{m} \mathbb{A}^{m} \sum_{\ell=m}^{q} \frac{1}{(\ell-m) !}\left(x_{I_{R}}-\frac{a}{2}\right)^{\ell-m} \partial_{x}^{\ell} \boldsymbol{u}\left(\frac{a}{2}, t_{n}\right) \\
& +\mathcal{O}\left(\Delta x^{q+1}\right)+\mathcal{O}\left(\Delta x^{K+1}\right) .
\end{aligned}
$$

Summing $\mathcal{L}_{0, \boldsymbol{u}}^{0}$ and the terms $\mathcal{L}_{0, \boldsymbol{u}}^{m}$ yields:

$$
\mathcal{L}_{0, \boldsymbol{u}}\left(x_{I_{R}}, t_{n}\right)=\sum_{m=1}^{K} \mathbb{A}^{m} \Delta t^{m} \sum_{\ell=0}^{q} \varepsilon_{\ell, m} \partial_{x}^{\ell} \boldsymbol{u}\left(\frac{a}{2}, t_{n}\right)+\mathcal{O}\left(\Delta x^{\min (K+1, q)}\right),
$$


where, for $m=1, \ldots, K$ and $\ell=0, \ldots, q$, the parameter $\varepsilon_{\ell, m}$ is defined as

$$
\varepsilon_{\ell, m}=\frac{(-1)^{m}}{m !(\ell-m) !}\left(x_{I_{R}}-\frac{a}{2}\right)^{\ell-m} \delta_{\ell \geq m}+\sum_{s=-K / 2}^{K / 2} \nu_{K, m, s} \frac{1}{\ell ! \Delta x^{m}}\left(x_{I_{R}}-\frac{a}{2}+s \Delta x\right)^{\ell},
$$

with $\delta_{\ell \geq m}=0$ if $\ell<m$ and $\delta_{\ell \geq m}=1$ else. Expanding the second right-hand side term using the binomial expansion entails

$$
\varepsilon_{\ell, m}=\frac{(-1)^{m}}{m !(\ell-m) !}\left(x_{I_{R}}-\frac{a}{2}\right)^{\ell-m} \delta_{\ell \geq m}+\sum_{j=0}^{\ell} \frac{\Delta x^{j-m}}{j !(\ell-j) !}\left(x_{I_{R}}-\frac{a}{2}\right)^{\ell-j} \sum_{s=-K / 2}^{K / 2} \nu_{K, m, s} s^{j} .
$$

For $0 \leq j \leq K$ then (14) implies that it holds:

$$
\frac{(-1)^{m}}{m !(\ell-m) !}\left(x_{I_{R}}-\frac{a}{2}\right)^{\ell-m} \delta_{\ell \geq m}+\sum_{j=0}^{\min (\ell, K)} \frac{\Delta x^{j-m}}{j !(\ell-j) !}\left(x_{I_{R}}-\frac{a}{2}\right)^{\ell-j} \sum_{s=-K / 2}^{K / 2} \nu_{K, m, s} s^{j}=0 .
$$

Therefore, if $\ell \leq K$ then $\varepsilon_{\ell, m}=0$ and when $\ell>K$ then (A.13) reduces to

$$
\varepsilon_{\ell, m}=\sum_{j=K+1}^{\ell} \frac{\Delta x^{j-m}}{j !(\ell-j) !}\left(x_{I_{R}}-\frac{a}{2}\right)^{\ell-j} \sum_{s=-K / 2}^{K / 2} \nu_{K, m, s} s^{j}=\mathcal{O}\left(\Delta x^{\ell-m}\right) \sum_{j=K+1}^{\ell} \sum_{s=-K / 2}^{K / 2} \frac{\nu_{K, m, s} s^{j}}{j !(\ell-j) !} .
$$

For all $(\ell, m)$ the results for these two cases can be summarized as follows:

$$
\varepsilon_{\ell, m}=\mathcal{O}\left(\Delta x^{\ell-m}\right) \delta_{\ell>K}
$$

This allows to conclude the proof of the first part of Property 1 since, using (A.16) in (A.12), then for $q=3$ and $q=5$ one gets:

$$
\mathcal{L}\left(x_{I_{R}}, t_{n}\right)=\mathcal{O}\left(\Delta x^{\min (K+1, q)}\right)
$$

and the same holds for $x_{I_{R}}$, or any other irregular point.

Appendix A.2. Estimation of $\mathcal{L}_{0, \Lambda_{r}^{i}}$

$\mathcal{L}_{0, \Lambda_{r}^{i}}$ is defined in (53) and can be expressed using Approximation 2 as

$$
\mathcal{L}_{0, \Lambda_{r}^{i}}\left(t_{n}\right)=\Lambda_{r}^{i}\left(t_{n+1}\right)-\Lambda_{r}^{i}\left(t_{n}\right)-\frac{\alpha_{r}^{2}}{\omega_{r} \rho_{m}} \sum_{m=0}^{q_{I}-2} \gamma_{m} \sum_{w=0}^{q_{D}} \beta_{w}\left(\widetilde{\left\langle\partial_{x}^{2} p\right\rangle_{a}}\right)\left(t_{n-m-w}\right)\left(\kappa_{r}^{i}\right)^{n-m},
$$

where $\left\langle\widetilde{\partial_{x}^{2} p}\right\rangle_{a}\left(t_{s}\right)$ denotes the trace value obtained when replacing the numerical values $\left(\boldsymbol{u}^{s}, \boldsymbol{\Lambda}^{s}, \boldsymbol{Z}^{s}\right)$ by the exact continuous solutions in (46). This term writes:

$\widetilde{\left\langle\partial_{x}^{2} p\right\rangle_{a}}\left(t_{n-m-w}\right)=\frac{1}{2}\left[\left(\mathbb{I}_{q_{U}}+\left(\mathbb{C}_{+}^{q}\right)^{-1} \mathbb{C}_{-}^{q}\right)\left(\mathbb{M}^{q}\right)^{-1} \mathcal{U}\left(t_{n-m-w}\right)+\left(\left(\mathbb{M}^{q}\right)^{-1} \mathbb{F}^{q}+\left(\mathbb{C}_{+}^{q}\right)^{-1} \mathbb{G}^{q}\right) \mathbb{Q}^{q} \boldsymbol{Z}\left(t_{n-m-w}\right)\right][3]$, 
Using (45), (28) and (29), one gets

$$
\begin{aligned}
\left\langle\widetilde{\partial_{x}^{2} p}\right\rangle_{a}\left(t_{n-m-w}\right) & \left.=\left\langle\partial_{x}^{2} p\right\rangle_{a}\right)\left(t_{n-m-w}\right) \\
& +\frac{1}{2}\left[-\left(\mathbb{C}_{+}^{q}\right)^{-1} \mathbb{G}^{q}\left(\mathbb{R}_{-}^{q}\left(t_{n-m-w}\right)-\mathbb{R}_{+}^{q}\left(t_{n-m-w}\right)\right)+\left(\mathbb{M}^{q}\right)^{-1} \Delta\left(t_{n-m-w}\right)\right][3] \\
& -\frac{1}{2}\left[\left(\mathbb{I}_{q_{U}}+\left(\mathbb{C}_{+}^{q}\right)^{-1} \mathbb{C}_{-}^{q}\right)\left(\mathbb{M}^{q}\right)^{-1} \mathcal{O}\left(\Delta x^{q+1}\right)\right][3]
\end{aligned}
$$

Symbolic computations are then performed using Maple to estimate the leading contributions of the entries of the vectors in (A.4). Doing so for $q=3$ or 5 yields:

$$
\left\{\begin{array}{l}
{\left[-\left(\mathbb{C}_{+}^{q}\right)^{-1} \mathbb{G}^{q}\left(\mathbb{R}_{-}^{q}\left(t_{n-m-w}\right)-\mathbb{R}_{+}^{q}\left(t_{n-m-w}\right)\right)+\left(\mathbb{M}^{q}\right)^{-1} \Delta\left(t_{n-m-w}\right)\right][3]=\mathcal{O}\left(\Delta x^{q-1}\right),} \\
{\left[\left(\mathbb{I}_{q_{U}}+\left(\mathbb{C}_{+}^{q}\right)^{-1} \mathbb{C}_{-}^{q}\right)\left(\mathbb{M}^{q}\right)^{-1} \mathcal{O}\left(\Delta x^{q+1}\right)\right][3]=\mathcal{O}\left(\Delta x^{q}\right) .}
\end{array}\right.
$$

Using (A.20) and (A.21) combined with (44) in (A.18) leads to the third estimate of Property 1:

$$
\mathcal{L}_{0, \Lambda_{r}^{i}}\left(t_{n}\right)=\mathcal{O}\left(\Delta x^{\min \left(q_{I}, q_{D}+1\right)}\right)+\mathcal{O}\left(\Delta x^{q-1}\right) \sum_{m=0}^{q_{I}-2}\left(\kappa_{r}^{i}\right)^{n-m} \quad\left(\text { for } i=1,2 \text {, and } r=1, \ldots, N_{R}\right) .
$$

Appendix A.3. Estimation of $\mathcal{L}_{0, Z}$

$\mathcal{L}_{0, \boldsymbol{Z}}$ is defined in (53). To compute it, we have to notice, using trigonometric formulas, that

$$
\mathbb{S}\left(t_{n+1}\right)\left(\left\{\left(\kappa_{r}^{1}\right)^{n-m}\right\}_{r},\left\{\left(\kappa_{r}^{2}\right)^{n-m}\right\}_{r}\right)=-\left(\left\{\omega_{r}(m+1) \mathcal{O}(\Delta t)\right\}_{r},\left\{\omega_{r} \mathcal{O}(1)\right\}_{r}\right) .
$$

Using (A.22) and (A.23), one gets the last estimate of Property 1

$$
\mathcal{L}_{0, \boldsymbol{Z}}\left(t_{n}\right)=\left(\left\{\mathcal{O}\left(\Delta x^{\min \left(q, q_{I}, q_{D}+1\right)}\right)\right\}_{1 \leq j \leq N_{R}},\left\{\mathcal{O}\left(\Delta x^{\min \left(q-1, q_{I}, q_{D}+1\right)}\right)\right\}_{N_{R}+1 \leq j \leq 2 N_{R}}\right) .
$$

\section{Appendix B. Proof of Property 2}

Due to the expression of $\mathcal{H}_{L}$ in $(36)$ then $\mathcal{L}_{\boldsymbol{u}}\left(x_{I_{L}}, t_{n}\right)$ defined in (55) can be expressed as

$$
\mathcal{L}_{\boldsymbol{u}}\left(x_{I_{L}}, t_{n}\right)=\mathcal{L}_{0, \boldsymbol{u}}\left(x_{I_{L}}, t_{n+1}\right)-\mathcal{H}_{L}\left(\mathcal{L}_{0, \boldsymbol{u}}\left(\cdot, t_{n}\right), \frac{1}{\Delta t} \mathcal{L}_{0, \boldsymbol{Z}}\left(t_{n}\right)\right)
$$

and the same holds for the right side.

Thus, we aim at evaluating the order of $\mathcal{H}_{L}\left(\mathcal{L}_{0, \boldsymbol{u}}\left(\cdot, t_{n}\right), \frac{1}{\Delta t} \mathcal{L}_{0, \boldsymbol{Z}}\left(t_{n}\right)\right)$ and $\mathcal{H}_{R}\left(\mathcal{L}_{0, \boldsymbol{u}}\left(\cdot, t_{n}\right), \frac{1}{\Delta t} \mathcal{L}_{0, \boldsymbol{Z}}\left(t_{n}\right)\right)$. To do so, symbolic computations are then performed using Maple to estimate the leading contributions of the entries of the vectors in $\mathcal{H}_{L}$ (36) and $\mathcal{H}_{R}$ (38). Doing so provides, for $q=3$ or 5:

$$
\left\{\begin{array}{l}
\mathbb{T}_{I_{L}+s}^{q}\left(-\frac{a}{2}\right)\left(\mathbb{M}^{q}\right)^{-1}=\mathcal{O}(1), \\
\mathbb{T}_{I_{L}+s}^{q}\left(-\frac{a}{2}\right)\left(\mathbb{M}^{q}\right)^{-1} \mathbb{F}^{q} \mathbb{Q}^{q}=\mathbb{B}_{\Delta x}, \\
\mathbb{T}_{I_{R}+s}^{q}\left(\frac{a}{2}\right)\left(\mathbb{C}_{+}^{q}\right)^{-1} \mathbb{C}_{-}^{q}\left(\mathbb{M}^{q}\right)^{-1}=\mathcal{O}(1), \\
\mathbb{T}_{I_{R}+s}^{q}\left(\frac{a}{2}\right)\left(\mathbb{C}_{+}^{q}\right)^{-1} \mathbb{G}^{q} \mathbb{Q}^{q}=\mathbb{B}_{\Delta x},
\end{array}\right.
$$


with $\mathbb{B}_{\Delta x}$ being a $2 \times 2 N_{R}$ matrix defined by

$$
\mathbb{B}_{\Delta x}[i, j]= \begin{cases}\mathcal{O}\left(\Delta x^{2}\right) & \text { for } i \in\left\{1, \ldots, N_{R}\right\} \text { and } j \in\left\{N_{R}+1, \ldots, 2 N_{R}\right\}, \\ \mathcal{O}(\Delta x) & \text { for } i \in\left\{N_{R}+1, \ldots, 2 N_{R}\right\} \text { and } j \in\left\{1, \ldots, N_{R}\right\}, \\ 0 & \text { else. }\end{cases}
$$

The term $\mathcal{L}_{0, \boldsymbol{u}}\left(x_{i}, t_{n}\right)$ is determined in Property 1 for $x_{i}$ an irregular point. For a regular point, the error analysis of a ADER- $K$ scheme in a homogeneous medium can be used and $\mathcal{L}_{0, \boldsymbol{u}}\left(x_{i}, t_{n}\right)=\mathcal{O}\left(\Delta x^{K+1}\right) . \mathcal{L}_{0, \boldsymbol{Z}}\left(t_{n}\right)$ is also determined in Property 1. Using these results combined with (B.2) in (36) and (38), we finally obtain

$$
\mathcal{L}_{u}\left(x_{I_{L}}, t_{n}\right)=\mathcal{O}\left(\Delta x^{\min \left(K+1, q, q_{I}, q_{D+1}\right)}\right),
$$

and the same holds for $x_{I_{R}}$, or any other irregular point. Therefore, the final estimate given by Property 2 writes:

$$
\mathcal{E}\left(t_{n}\right)=\mathcal{O}\left(\Delta x^{\min \left(K, q-1, q_{I}-1, q_{D}\right)}\right) .
$$

\section{Appendix C. Semi-analytical solution for a plane wave on a plane interface}

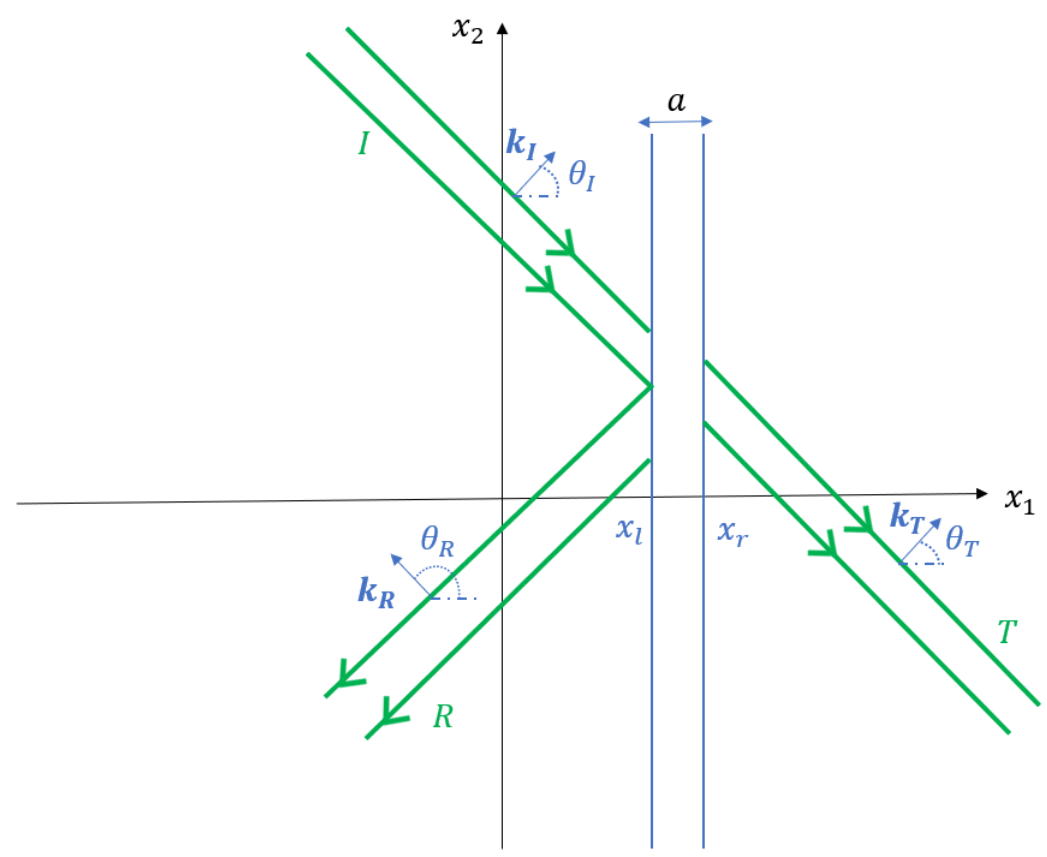

Figure C.13: Incident plane wave (I) illuminating the enlarged interface and leading to reflected (R) and transmitted $(\mathrm{T})$ plane waves.

We consider an incident plane wave at an angle $\theta_{I}$ with the horizontal axis and an enlarged interface located between the physical points $x_{1}=x_{l}$ and $x_{1}=x_{r}$, see Figure C.13. The initial conditions are given in (60). To calculate the solution to the $2 \mathrm{D}$ problem (57) in this configuration, we consider its frequency-domain formulation (3). The wavefield solution $\hat{\boldsymbol{u}}(\boldsymbol{x}, \omega)$ 
is then decomposed into incident $\hat{\boldsymbol{u}}_{I}$, reflected $\hat{\boldsymbol{u}}_{R}$ and transmitted $\hat{\boldsymbol{u}}_{T}$ waves, ie $\hat{\boldsymbol{u}}=\hat{\boldsymbol{u}}_{I}+\hat{\boldsymbol{u}}_{R}+$ $\hat{\boldsymbol{u}}_{T}$. It is assumed that these are plane waves that write

$$
\left\{\begin{aligned}
& \hat{\boldsymbol{u}}_{I}=\left(\begin{array}{c}
-\rho_{m} \\
-\cos \theta_{I} / c_{m} \\
-\sin \theta_{I} / c_{m}
\end{array}\right) \exp \left(-\boldsymbol{k}_{I} \cdot\left(\boldsymbol{x}-\boldsymbol{x}_{s}\right)\right) \hat{g}(\omega), \\
& \hat{\boldsymbol{u}}_{R}=\left(\begin{array}{c}
-\rho_{m} \\
-\cos \theta_{R} / c_{m} \\
-\sin \theta_{R} / c_{m}
\end{array}\right) \exp \left(-\boldsymbol{k}_{R} \cdot\left(\boldsymbol{x}-\boldsymbol{x}_{s}\right)\right) \hat{g}(\omega) R(\omega), \\
& \hat{\boldsymbol{u}}_{T}=\left(\begin{array}{c}
-\rho_{m} \\
-\cos \theta_{T} / c_{m} \\
-\sin \theta_{T} / c_{m}
\end{array}\right) \exp \left(-\boldsymbol{k}_{T} \cdot\left(\boldsymbol{x}-\boldsymbol{x}_{s}\right)\right) \hat{g}(\omega) T(\omega),
\end{aligned}\right.
$$

with $\boldsymbol{k}_{I}, \boldsymbol{k}_{R}$ and $\boldsymbol{k}_{T}$ being the corresponding wavevectors that are of norm $\omega / c_{m}$ and whose direction is normal to the wave fronts. Using the jump conditions in (3) and introducing the following parameters

$$
\left\{\begin{array}{l}
\alpha_{1}(\omega)=\frac{1}{2 c_{m}^{2}}\left(\left(C_{11}+h \mathcal{D}(\omega)\right) \cos \left(\theta_{R}\right)^{2}-C_{12} \sin \theta_{R} \cos \theta_{R}+\left(C_{22}+h \mathcal{D}(\omega)\right) \sin \left(\theta_{R}\right)^{2}\right) \\
\alpha_{2}(\omega)=\frac{1}{2 c_{m}^{2}}\left(\left(C_{11}+h \mathcal{D}(\omega)\right) \cos \left(\theta_{T}\right)^{2}-C_{12} \sin \theta_{T} \cos \theta_{R}+\left(C_{22}+h \mathcal{D}(\omega)\right) \sin \left(\theta_{T}\right)^{2}\right) \\
\alpha_{3}(\omega)=\frac{1}{2 c_{m}^{2}}\left(\left(C_{11}+h \mathcal{D}(\omega)\right) \cos \left(\theta_{I}\right)^{2}-C_{12} \sin \theta_{I} \cos \theta_{R}+\left(C_{22}+h \mathcal{D}(\omega)\right) \sin \left(\theta_{I}\right)^{2}\right) \\
\beta_{1}=\frac{\rho_{m}}{2 c_{m}}\left(B_{1} \cos \theta_{R}+B_{2} \sin \theta_{R}\right) \\
\beta_{2}=\frac{\rho_{m}}{2 c_{m}}\left(B_{1} \cos \theta_{T}+B_{2} \sin \theta_{T}\right), \\
\beta_{3}=\frac{\rho_{m}}{2 c_{m}}\left(B_{1} \cos \theta_{I}+B_{2} \sin \theta_{I}\right), \\
\delta_{R}(\omega)=\exp \left(\mathrm{i} \frac{\omega}{c_{m}}\left(x_{l} \cos \theta_{I}-x_{l} \cos \theta_{R}\right)\right) \\
\delta_{T}(\omega)=\exp \left(\mathrm{i} \frac{\omega}{c_{m}}\left(x_{l} \cos \theta_{I}-x_{r} \cos \theta_{T}\right)\right)
\end{array}\right.
$$

then we get the following system concerning the reflected and transmitted waves:

$$
\left\{\begin{array}{l}
\left(\frac{\cos \theta_{R}}{c_{m}}-\mathrm{i} \omega \alpha_{1}\right) \delta_{R}(\omega) R(\omega)-\left(\frac{\cos \theta_{T}}{c_{m}}+\mathrm{i} \omega \alpha_{2}\right) \delta_{T}(\omega) T(\omega)=-\frac{\cos \theta_{I}}{c_{m}}+\mathrm{i} \omega \alpha_{3} \\
\left(\rho_{m}-\mathrm{i} \omega \beta_{1}\right) \delta_{R}(\omega) R(\omega)-\left(\rho_{m}+\mathrm{i} \omega \beta_{2}\right) \delta_{T}(\omega) T(\omega)=-\rho_{m}+\mathrm{i} \omega \beta_{3} .
\end{array}\right.
$$


Introducing the parameters

$$
\left\{\begin{array}{l}
Z_{1}=\rho_{m} c_{m} \cos \theta_{I}, \\
Z_{2}=\rho_{m} c_{m} \cos \theta_{T}, \\
\mathcal{C}_{1}(\omega)=c_{m}^{2}\left(\rho_{m} \alpha_{1}(\omega)+\rho_{m} \alpha_{2}(\omega)+\frac{\beta_{2}}{c_{m}} \cos \theta_{I}-\frac{\beta_{1}}{c_{m}} \cos \theta_{T}\right), \\
\mathcal{C}_{2}(\omega)=c_{m}^{2}\left(-\rho_{m} \alpha_{3}(\omega)-\rho_{m} \alpha_{2}(\omega)+\frac{\beta_{2}}{c_{m}} \cos \theta_{I}+\frac{\beta_{3}}{c_{m}} \cos \theta_{T}\right), \\
\mathcal{C}_{3}(\omega)=c_{m}^{2}\left(\rho_{m} \alpha_{1}(\omega)-\rho_{m} \alpha_{3}(\omega)-\frac{\beta_{3}}{c_{m}} \cos \theta_{I}+\frac{\beta_{1}}{c_{m}} \cos \theta_{R}\right), \\
\mathcal{M}_{1}(\omega)=c_{m}^{2}\left(\alpha_{2}(\omega) \beta_{1}-\alpha_{1}(\omega) \beta_{2}\right), \\
\mathcal{M}_{2}(\omega)=c_{m}^{2}\left(\alpha_{3}(\omega) \beta_{2}-\alpha_{2}(\omega) \beta_{3}\right), \\
\mathcal{M}_{3}(\omega)=c_{m}^{2}\left(\alpha_{1}(\omega) \beta_{3}-\alpha_{3}(\omega) \beta_{1}\right),
\end{array}\right.
$$

we get the reflection coefficient and the transmission coefficient

$$
\left\{\begin{array}{l}
R(\omega)=\frac{Z_{1}-Z_{2}+i \omega \mathcal{C}_{2}+\omega^{2} \mathcal{M}_{2}}{Z_{1}+Z_{2}+i \omega \mathcal{C}_{1}+\omega^{2} \mathcal{M}_{1}} \exp \left(-i \frac{\omega}{c_{m}}\left(x_{l} \cos \theta_{I}-x_{l} \cos \theta_{R}\right)\right), \\
T(\omega)=\frac{2 Z_{1}+i \omega \mathcal{C}_{3}+\omega^{2} \mathcal{M}_{3}}{Z_{1}+Z_{2}+i \omega \mathcal{C}_{1}+\omega^{2} \mathcal{M}_{1}} \exp \left(-i \frac{\omega}{c_{m}}\left(x_{l} \cos \theta_{I}-x_{r} \cos \theta_{T}\right)\right),
\end{array}\right.
$$

with $\theta_{R}=\pi / 2+\theta_{I}$ and $\theta_{T}=\theta_{I}$. These expressions used in (C.1) give the sought expression for $\hat{\boldsymbol{u}}(\boldsymbol{x}, \omega)$. An inverse discrete Fourier transform in time provides the semi-analytical timedomain solution considered in Section 5. It can be shown that the 1D semi-analytical solution is recovered when setting $\theta_{I}=0, x_{2}=0$ and $v_{2}=0$ in the $2 \mathrm{D}$ solution.

Remark 7. When the enlarged interface is tilted of an angle $-\phi$ with the horizontal axis then the calculation of the associated scattering coefficients follows the same lines in the rotated coordinate system $(\zeta, \eta)=\left(\cos \phi x_{1}-\sin \phi x_{2}, \sin \phi x_{1}+\cos \phi x_{2}\right)$. A rotation of angle $-\phi i s$ then necessary after the inverse discrete Fourier transform to express the vectorial field $\boldsymbol{v}$ in the basis $\left(x_{1}, x_{2}\right)$.

\section{Appendix D. Semi-analytical solution for a plane wave on a circular interface}

We consider an incident plane wave at an angle $\theta_{I}$ with the horizontal axis and an enlarged circular interface defined by two circles of centers $\left(x_{c}, y_{c}\right)$ and radii $a^{-}$and $a^{+}$, with $a^{-}>$ $a^{+}$. The initial conditions are given in (60). The approach presented here applies to the particular interface parameter values $B_{2}=C_{12}=\alpha_{0}=0$, the other parameters being arbitrary. The method employed in [15] for fluid-solid circular interface is applied here: to calculate the solution, we consider its frequency-domain formulation (3). The wavefield solution $\hat{\boldsymbol{u}}(\boldsymbol{x}, \omega)$ is then decomposed into incident, reflected and transmitted waves. They are written on a truncated basis of Bessel functions using the Jacobi-Anger decomposition and the associated 
diffraction coefficients are derived from the jump conditions. We start by introducing the harmonic acoustic potential $\hat{\Phi}$ such that

$$
\hat{p}=-\mathrm{i} \omega \rho_{m} \hat{\Phi}, \quad \hat{v}_{\nu}=\partial_{r} \hat{\Phi}, \quad \hat{v}_{\tau}=\frac{1}{r} \partial_{\phi} \hat{\Phi}
$$

The harmonic potential of the incident plane wave is:

$$
\hat{\Phi}_{I}\left(x_{1}, x_{2}, \omega\right)=\exp \left(-\frac{\mathrm{i} \omega}{c_{m}}\left(x_{1} \cos \theta_{I}+x_{2} \sin \theta_{I}\right)\right) \frac{\hat{g}(\omega)}{\mathrm{i} \omega} .
$$

We introduce a polar coordinates system $(r, \phi)$ such as $x_{1}=x_{c}+r \cos \phi$ and $x_{2}=y_{c}+r \sin \phi$, so the above potential reads:

$$
\hat{\Phi}_{I}\left(x_{1}, x_{2}, \omega\right)=F S \exp (-\mathrm{i} k r \cos \alpha)
$$

with $F=\hat{g}(\omega) / \mathrm{i} \omega, S=\exp \left(-\mathrm{i} k\left(x_{c} \cos \theta_{I}+y_{c} \sin \theta_{I}\right), \alpha=\phi-\theta_{I}\right.$ and $k=\omega / c_{m}$. The Bessel functions of the first-kind $\mathcal{J}_{n}$ satisfy the Jacobi-Anger expansion, see e.g. [10]:

$$
\exp (-\mathrm{i} r \cos \alpha)=\sum_{n=0}^{\infty} \epsilon_{n}(-\mathrm{i})^{n} \cos (n \alpha) \mathcal{J}_{n}(r)
$$

with $\epsilon_{n}=1$ if $n=0,2$ else. From (D.3) and (D.4), we therefore express the potential $\hat{\Phi}_{I}$ as:

$$
\hat{\Phi}_{I}\left(x_{1}, x_{2}, \omega\right)=F S \sum_{n=0}^{\infty} \epsilon_{n}(-\mathrm{i})^{n} \cos (n \alpha) \mathcal{J}_{n}(k r) .
$$

To satisfy the Sommerfeld condition, which is satisfied by the radiated wavefield at infinity in the classical acoustic medium, the harmonic acoustic potential $\hat{\Phi}_{R}$ of the reflected wave is written on the basis of Hankel functions of the second-kind $\mathcal{H}_{n}$. To prevent singularities from occurring at $r=0$, the harmonic potential $\hat{\Phi}_{T}$ of transmitted waves is written on the basis of the Bessel functions of the first-kind, i.e.

$$
\hat{\Phi}_{R}\left(x_{1}, x_{2}, \omega\right)=\sum_{n=0}^{\infty} R_{n} \cos (n \alpha) \mathcal{H}_{n}(k r), \quad \hat{\Phi}_{T}\left(x_{1}, x_{2}, \omega\right)=\sum_{n=0}^{\infty} T_{n} \cos (n \alpha) \mathcal{J}_{n}(k r),
$$

where $R_{n}$ and $T_{n}$ are the coefficients of reflexion and transmission that have to be determined. The velocity $\hat{v}=\left(\hat{v}_{\nu}, \hat{v}_{\tau}\right)^{\top}$ and the pressure $\hat{p}$ fields are deduced from the potential $\hat{\Phi}$ using (D.3). From (D.1), (D.5) and (D.6), we deduce the components of the incident, reflected and 
transmitted waves as follows:

$$
\begin{aligned}
& \left\{\begin{array}{l}
\hat{p}_{I}=-\mathrm{i} \omega \rho_{m} F S \sum_{n=0}^{\infty} \epsilon_{n}(-\mathrm{i})^{n} \cos (n \alpha) \mathcal{J}_{n}(k r), \\
\hat{v}_{I \nu}=k F S \sum_{n=0}^{\infty} \epsilon_{n}(-\mathrm{i})^{n} \cos (n \alpha) \mathcal{J}_{n}^{\prime}(k r), \\
\hat{v}_{I \tau}=-\frac{1}{r} F S \sum_{n=0}^{\infty} \epsilon_{n}(-\mathrm{i})^{n} n \sin (n \alpha) \mathcal{J}_{n}(k r),
\end{array}\right. \\
& \left\{\begin{array}{l}
\hat{p}_{R}=-\mathrm{i} \omega \rho_{m} \sum_{n=0}^{\infty} R_{n} \cos (n \alpha) \mathcal{H}_{n}(k r), \\
\hat{v}_{R \nu}=k \sum_{n=0}^{\infty} R_{n} \cos (n \alpha) \mathcal{H}_{n}^{\prime}(k r) \\
\hat{v}_{R \tau}=-\frac{1}{r} \sum_{n=0}^{\infty} R_{n} n \sin (n \alpha) \mathcal{H}_{n}(k r),
\end{array}\right. \\
& \left\{\begin{array}{l}
\hat{p}_{T}=-\mathrm{i} \omega \rho_{m} \sum_{n=0}^{\infty} T_{n} \cos (n \alpha) \mathcal{J}_{n}(k r), \\
\hat{v}_{T \nu}=k \sum_{n=0}^{\infty} T_{n} \cos (n \alpha) \mathcal{J}_{n}^{\prime}(k r), \\
\hat{v}_{T \tau}=-\frac{1}{r} \sum_{n=0}^{\infty} T_{n} n \sin (n \alpha) \mathcal{J}_{n}(k r) .
\end{array}\right.
\end{aligned}
$$

In the case where $B_{2}=C_{12}=\alpha_{0}=0$ an identification is possible, then the coefficients $R_{n}$ and $T_{n}$ are deduced from the jump conditions in (57), which for all $0 \leq \phi \leq 2 \pi$ read:

$$
\hat{p}_{T}\left(a^{+}, \phi\right)-\left(\hat{p}_{I}+\hat{p}_{R}\right)\left(a^{-}, \phi\right)=\frac{B_{1}}{2}\left(\partial_{r} \hat{p}_{T}\left(a^{+}, \phi\right)+\left(\partial_{r} \hat{p}_{I}+\partial_{r} \hat{p}_{R}\right)\left(a^{-}, \phi\right)\right),
$$

and

$$
\begin{aligned}
\hat{v}_{T \nu}\left(a^{+}, \phi\right)-\left(\hat{v}_{I \nu}+\right. & \left.\hat{v}_{R \nu}\right)\left(a^{-}, \phi\right)=\frac{C_{11}}{2}\left(\partial_{r} \hat{v}_{T \nu}\left(a^{+}, \phi\right)+\left(\partial_{r} \hat{v}_{I \nu}+\partial_{r} \hat{v}_{R \nu}\right)\left(a^{-}, \phi\right)\right) \\
+ & \frac{C_{22}}{2}\left(\frac{1}{a^{+}} \partial_{\phi} \hat{v}_{T \tau}\left(a^{+}, \phi\right)+\frac{1}{a^{-}}\left(\partial_{\phi} \hat{v}_{I \tau}+\partial_{\phi} \hat{v}_{R \tau}\right)\left(a^{-}, \phi\right)\right) \\
& +\frac{k^{2} h \mathcal{D}(\omega)}{2}\left(\hat{p}_{T}\left(a^{+}, \phi\right)+\left(\hat{p}_{I}+\hat{p}_{R}\right)\left(a^{-}, \phi\right)\right) .
\end{aligned}
$$


From there, one obtains the following system satisfied by the coefficients $R_{n}$ and $T_{n}$ for all $n \geq 0$ :

$$
\left\{\begin{array}{l}
{\left[k \mathcal{J}_{n}^{\prime}\left(k a^{+}\right)-\frac{C_{11}}{2} k^{2} \mathcal{J}_{n}^{\prime \prime}\left(k a^{+}\right)+\frac{C_{22}}{2}\left(\frac{n}{a^{+}}\right)^{2} \mathcal{J}_{n}\left(k a^{+}\right)-k^{2} \frac{h}{2} \mathcal{D}(\omega) \mathcal{J}_{n}\left(k a^{+}\right)\right] T_{n}} \\
+\left[-k \mathcal{H}_{n}^{\prime}\left(k a^{-}\right)-\frac{C_{11}}{2} k^{2} \mathcal{H}_{n}^{\prime \prime}\left(k a^{-}\right)+\frac{C_{22}}{2}\left(\frac{n}{a^{-}}\right)^{2} \mathcal{H}_{n}\left(k a^{-}\right)-k^{2} \frac{h}{2} \mathcal{D}(\omega) \mathcal{H}_{n}\left(k a^{-}\right)\right] R_{n} \\
=F S \epsilon_{n}(-\mathrm{i})^{n}\left[k \mathcal{J}_{n}^{\prime}\left(k a^{-}\right)+\frac{C_{11}}{2} k^{2} \mathcal{J}_{n}^{\prime \prime}\left(k a^{-}\right)-\frac{C_{22}}{2}\left(\frac{n}{a^{-}}\right)^{2} \mathcal{J}_{n}\left(k a^{-}\right)+k^{2} \frac{h}{2} \mathcal{D}(\omega) \mathcal{J}_{n}\left(k a^{-}\right)\right] \\
{\left[\mathcal{J}_{n}\left(k a^{+}\right)-\frac{B_{1}}{2} k \mathcal{J}_{n}^{\prime}\left(k a^{+}\right)\right] T_{n}+\left[-\mathcal{H}_{n}\left(k a^{-}\right)-\frac{B_{1}}{2} k \mathcal{H}_{n}^{\prime}\left(k a^{-}\right)\right] R_{n}} \\
=F S \epsilon_{n}(-\mathrm{i})^{n}\left[\mathcal{J}_{n}\left(k a^{-}\right)+\frac{B_{1}}{2} k \mathcal{J}_{n}^{\prime}\left(k a^{-}\right)\right] .
\end{array}\right.
$$

In practice, one considers a finite number $N_{\text {Bessel }}$ of modes. The coefficients $R_{n}$ and $T_{n}$ are computed from the associated systems (D.8) and (D.7) is finally used to obtain the wavefield solution $\hat{\boldsymbol{u}}(\boldsymbol{x}, \omega)$ in the frequency domain. A discrete inverse Fourier transform in time yields the semi-analytical solution considered in Section 5.2.3.

[1] C. Bellis and B. Lombard. Simulating transient wave phenomena in acoustic metamaterials using auxiliary fields. Wave Motion, 86:175-194, March 2019.

[2] A. Bensoussan, J.-L. Lions, and G. Papanicolaou. Asymptotic Analysis for Periodic Structures. North-Holland, 1978.

[3] Marc Bonnet, Rémi Cornaggia, and Bojan B. Guzina. Microstructural topological sensitivities of the second-order macroscopic model for waves in periodic media. SIAM Journal on Applied Mathematics, 78(4):2057-2082, jan 2018.

[4] A. Bonnet-Bendhia, D. Drissi, and N. Gmati. Simulation of a muffler's transmission losses by a homogenized finite element method. Journal of Computational Acoustics, 12(3):447474, 2004.

[5] Joly P. Cassier M. and Kachanovska M. Mathematical models for dispersive electromagnetic waves: An overview. Computers $\&$ Mathematics with Applications, 74(11):2792-2830, 2017.

[6] Guillaume Chiavassa and Bruno Lombard. Time domain numerical modeling of wave propagation in 2D heterogeneous porous media. Journal of Computational Physics, 230(13):5288-5309, jun 2011.

[7] M. David, C. Pideri, and J.-J. Marigo. Homogenized interface model describing inhomogeneities located on a surface. Journal of Elasticity, 109(2):153-187, 2012.

[8] Bérangre Delourme. Modèles asymptotiques des interfaces fines et périodiques en électromagnétisme. PhD Thesis - Universit Pierre et Marie Curie - Paris VI, 2010. 
[9] Bojan B Guzina and Marc Bonnet. Small-inclusion asymptotic of misfit functionals for inverse problems in acoustics. Inverse Problems, 22(5):1761-1785, sep 2006.

[10] R. B. Harvey, P. M. Morse, and H. Feschbach. Methods of theoretical physics. The Mathematical Gazette, 39(327):80, feb 1955.

[11] B. Lombard. Modélisation numérique de la propagation et de la diffraction d'ondes mécaniques. Habilitation à Diriger des Recherches de l'Université Aix-Marseille 2, 2010.

[12] B. Lombard, A. Maurel, and J.-J. Marigo. Numerical modeling of the acoustic wave propagation across an homogenized rigid microstructure in the time domain. Journal of Computational Physics, 335:558-577, 2017.

[13] B. Lombard, J. Piraux, C. Gélis, and J. Virieux. Free and smooth boundaries in 2-d finite-difference schemes for transient elastic waves. Geophysical Journal International, 172(1):252-261, jan 2008.

[14] Bruno Lombard and Jol Piraux. How to incorporate the spring-mass conditions in finitedifference schemes. SIAM Journal of Scientific Computing, 24(4):1379-1407, 2003.

[15] Bruno Lombard and Jol Piraux. Numerical treatment of two-dimensional interfaces for acoustic and elastic waves. Journal of Computational Physics, 195(1):90-116, mar 2004.

[16] Bruno Lombard and Jol Piraux. Numerical modeling of elastic waves across imperfect contacts. SIAM Journal on Scientific Computing, 28(1):172-205, jan 2006.

[17] F. Lorcher and C.-D. Munz. Lax-wendroff-type schemes of arbitrary order in several space dimensions. IMA Journal of Numerical Analysis, 27(3):593-615, nov 2006.

[18] G. Ma, M. Yang, S. Xiao, Z. Yang, and P. Sheng. Acoustic metasurface with hybrid resonances. Nature Materials, 13:873-879, 2014.

[19] J.-J. Marigo and A. Maurel. Homogenization models for thin rigid structured surfaces and films. The Journal of the Acoustical Society of America, 140(1):260-273, 2016.

[20] J.-J. Marigo, A. Maurel, K. Pham, and A. Sbitti. Effective dynamic properites of a row of elastic inclusions: The case of scalar shear waves. Journal of Elasticity, 128(2):265-289, 2017.

[21] J.-J. Marigo and C. Pideri. The effective behaviour of elastic bodies containing microcracks or microholes localized on a surface. International Journal of Damage Mechanics, SAGE Publications, 20:1151-1177, 2011.

[22] K. Pham, A. Maurel, and J.-J. Marigo. Two scale homogenization of a row of locally resonant inclusions - the case of shear waves. Journal of the Mechanics and Physics of Solids, 106:80-94, 2017.

[23] J. Sanchez-Hubert and E. Sanchez-Palencia. Introduction aux Méthodes Asymptotiques et à l'Homogénéisation. Collection Mathématiques Appliquées pour la Maîtrise, 1992. 
[24] L. Schwan, O. Umnova, and C. Boutin. Sound absorption and reflection from a resonant metasurface: Homogenisation model with experimental validation. Wave Motion, 72:154$172,2017$.

[25] Thomas Schwartzkopff, Michael Dumbser, and Claus-Dieter Munz. Fast high order ADER schemes for linear hyperbolic equations. Journal of Computational Physics, 197(2):532539, jul 2004.

[26] X. Su, Z. Lu, and A.N. Norris. Elastic metasurfaces for splitting sv- and p-waves in elastic solids. Journal of Applied Physics, 123:091701, 2018.

[27] W. T. Vetterling W. H. Press, S. A. Teukolskyn and B. P. Flannery. Numerical Recipes in C: The Art of Scientific Computing. Cambridge University Press, 1992.

[28] C. Zhang and R. J. LeVeque. The immersed interface method for acoustic wave equations with discontinuous coefficients. Wave Motion, 25:237-263, 1997. 